\title{
A budding yeast model for human disease mutations in the EXOSC2 cap subunit of the RNA exosome complex
}

\author{
MARIA C. STERRETT, ${ }^{1,2,8}$ LIZ ENYENIHI ${ }^{2,8}$ SARA W. LEUNG, ${ }^{2}$ LAURIE HESS, ${ }^{2}$ SARAH E. STRASSLER, ${ }^{1,3}$ \\ DANIELA FARCHI, ${ }^{2}$ RICHARD S. LEE, ${ }^{2}$ ELISE S. WITHERS, ${ }^{2}$ ISAAC KREMSKY, ${ }^{4}$ RICHARD E. BAKER, ${ }^{5}$ \\ MUNIRA A. BASRAI, ${ }^{6}$ AMBRO VAN HOOF, ${ }^{7}$ MILO B. FASKEN, ${ }^{2}$ and ANITA H. CORBETT ${ }^{2}$ \\ ${ }^{1}$ Biochemistry, Cell and Developmental Biology Graduate Program, Emory University, Atlanta, Georgia 30322, USA \\ ${ }^{2}$ Department of Biology, Emory University, Atlanta, Georgia 30322, USA \\ ${ }^{3}$ Department of Biochemistry, Emory University, Atlanta, Georgia 30322, USA \\ ${ }^{4}$ Loma Linda University School of Medicine, Loma Linda, California 92350, USA \\ ${ }^{5}$ Department of Microbiology and Physiological Systems, University of Massachusetts Medical School, Worcester, Massachusetts 01655, USA \\ ${ }^{6}$ Genetics Branch, Center for Cancer Research, National Cancer Institute, National Institutes of Health, Bethesda, Maryland 20892, USA \\ ${ }^{7}$ Department of Microbiology and Molecular Genetics, University of Texas Health Science Center at Houston, Houston, Texas 77030, USA
}

\begin{abstract}
RNA exosomopathies, a growing family of diseases, are linked to missense mutations in genes encoding structural subunits of the evolutionarily conserved, 10-subunit exoribonuclease complex, the RNA exosome. This complex consists of a threesubunit cap, a six-subunit, barrel-shaped core, and a catalytic base subunit. While a number of mutations in RNA exosome genes cause pontocerebellar hypoplasia, mutations in the cap subunit gene EXOSC2 cause an apparently distinct clinical presentation that has been defined as a novel syndrome SHRF (short stature, hearing loss, retinitis pigmentosa, and distinctive facies). We generated the first in vivo model of the SHRF pathogenic amino acid substitutions using budding yeast by modéling pathogenic EXOSC2 missense mutations (p.Gly30Val and p.Gly198Asp) in the orthologous S. cerevisiae gene RRP4. The resulting rrp4 mutant cells show defects in cell growth and RNA exosome function. Consistent with altered RNA exosome function, we detect significant transcriptomic changes in both coding and noncoding RNAs in rrp4-G226D cells that model EXOSC2 p.Gly198Asp, suggesting defects in nuclear surveillance. Biochemical and genetic analyses suggest that the Rrp4 G226D variant subunit shows impaired interactions with key RNA exosome cofactors that modulate the function of the complex. These results provide the first in vivo evidence that pathogenic missense mutations present in EXOSC2 impair the function of the RNA exosome. This study also sets the stage to compare exosomopathy models to understand how defects in RNA exosome function underlie distinct pathologies.
\end{abstract}

Keywords: EXOSC2; exosomopathy; RNA decay/processing; RNA exosome; Rrp4

\section{INTRODUCTION}

The RNA exosome is an evolutionarily conserved, multisubunit riboexonuclease complex that plays multiple roles in RNA processing and decay. First identified in Saccharomyces cerevisiae in a screen for ribosomal RNA processing (rrp) mutants (Mitchell et al. 1996, 1997), the RNA exosome is essential in all systems studied thus far (Mitchell et al. 1997; Lorentzen et al. 2007; Hou et al. 2012; Lim et al. 2013; Pefanis et al. 2014). In addition to critical roles in generating mature ribosomal RNA, the

\footnotetext{
${ }^{8}$ Joint first authors.

Corresponding authors: acorbe2@emory.edu, mfasken@emory.edu

Article is online at http://www.rnajournal.org/cgi/doi/10.1261/rna. 078618.120 . Freely available online through the RNA Open Access option.
}

RNA exosome processes a variety of small noncoding RNAs (ncRNAs), including small nuclear RNAs (snRNAs), small nucleolar RNAs (snoRNAs), and transfer RNAs (tRNAs) (Allmang et al. 1999; van Hoof et al. 2000; Kilchert et al. 2016a; Fasken et al. 2020). Beyond processing numerous RNAs, the RNA exosome is also required for RNA decay and surveillance, including nuclear degradation of cryptic unstable transcripts (CUTs) in budding yeast that result from pervasive transcription (Wyers et al. 2005; Parker 2012; Schneider et al. 2012) and cytoplasmic RNA turnover of aberrant and nonfunctional mRNAs (Moore and Proudfoot 2009). Therefore, the RNA exosome has many far-reaching roles in vivo that affect nearly every class

(C) 2021 Sterrett et al. This article, published in RNA, is available under a Creative Commons License (Attribution 4.0 International), as described at http://creativecommons.org/licenses/by/4.0/. 
of RNA (Schneider et al. 2012; Schneider and Tollervey 2013).

This essential RNA processing/degradation machine is composed of nine structural subunits associated with a catalytic $3^{\prime}-5^{\prime}$ exo/endoribonuclease subunit (DIS3/DIS3L [human]; Dis3/Rrp44 [budding yeast]) (Mitchell et al. 1997; Makino et al. 2013). As illustrated in Figure 1A, the nine-subunit structural barrel is composed of an upper ring of three $\mathrm{S} 1 / \mathrm{KH}$ cap subunits (EXOSC1/2/3; Csl4/Rrp4/Rrp40) and a lower ring of six $\mathrm{PH}$-like subunits (EXOSC4/7/8/9/5/6; Rrp41/Rrp42/Rrp43/Rrp45/Rrp46/ Mtr3). Within the nucleus, an additional $3^{\prime}-5^{\prime}$ exonuclease termed EXOSC10/Rrp6 is also associated with the complex (Briggs et al. 1998; Wasmuth et al. 2014). Structural studies revealed conservation in the structural organization of the RNA exosome (Fig. 1B; Liu et al. 2006; Bonneau et al. 2009; Makino et al. 2013; Wasmuth et al. 2014; Zinder et al. 2016), suggesting evolutionary conservation not just of subunit sequence but of overall complex structure and organization.
As described above, a feature of the RNA exosome is the ability to both process and degrade numerous RNA targets. The specificity for this broad set of target transcripts is achieved, at least in part, through RNA exosome cofactors that associate with the complex via interactions with multiple subunits (Zinder and Lima 2017; Fasken et al. 2020). These cofactors are localized to both the nucleus and the cytoplasm, raising the possibility that these interactions facilitate targeting of distinct target transcripts in different cellular compartments. Nuclear RNA exosome cofactors have been extensively characterized in the budding yeast system, including the Rrp6 obligate binding partner Rrp47, the essential RNA helicase Mtr4, and Mpp6 (de la Cruz et al. 1998; LaCava et al. 2005; Schneider and Tollervey 2013; Zinder and Lima 2017). Structural studies of both the budding yeast and mammalian RNA exosome suggest Mpp6/MPH6, Rrp6/EXOSC10, and Rrp47/C1D can form composite sites that facilitate interactions between the complex and other cofactors, including Mtr4/ MTR4/MTREX (Schuch et al. 2014; Falk et al. 2017;
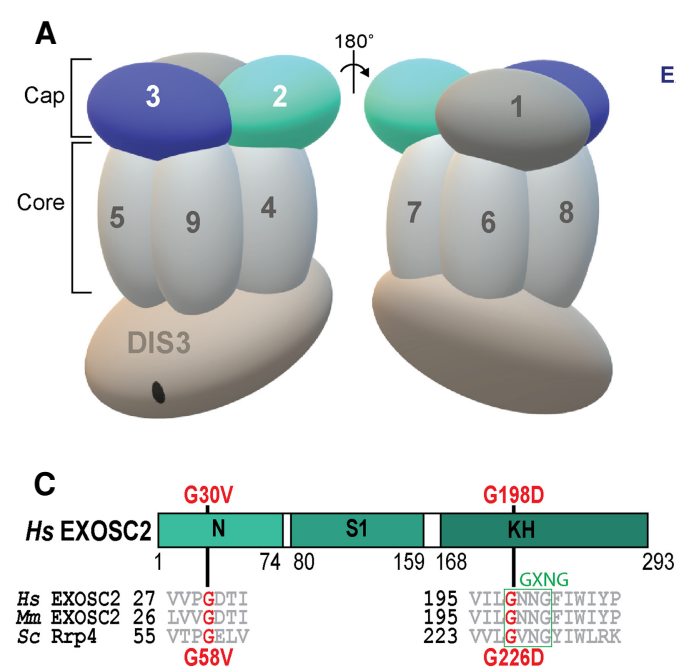
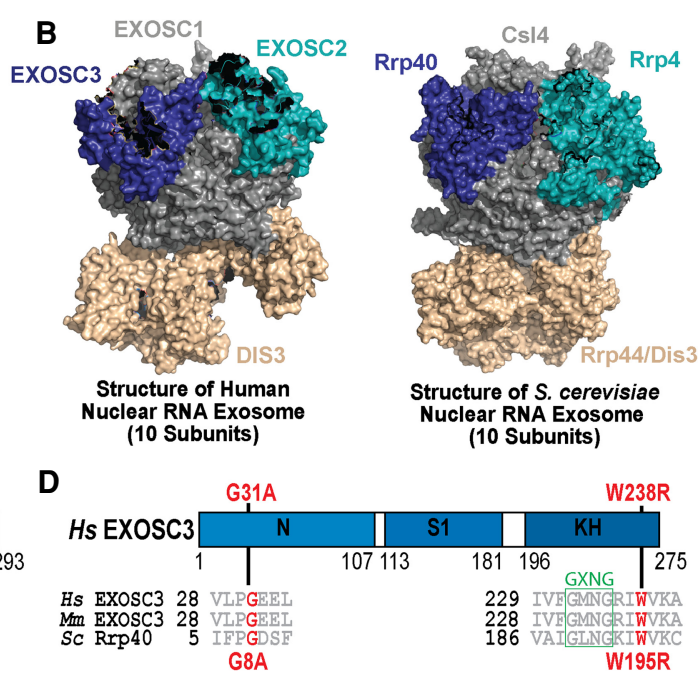

FIGURE 1. Overview of pathogenic amino acid substitutions in the human cap subunit EXOSC2 of the RNA exosome. (A) The RNA exosome is an evolutionary conserved ribonuclease complex composed of nine structural subunits (EXOSC1-9) and one catalytic subunit (DIS3) that form a "cap" and "core" ring-like structure. The three-subunit cap at the top of the complex is composed of EXOSC1/Cs/4 (Human/S. cerevisiae), EXOSC2/Rrp4, and EXOSC3/Rrp40. The six-subunit core is composed of EXOSC4/Rrp41, EXOSC5/Rrp46, EXOSC6/Mtr3, EXOSC7/Rrp42, EXOSC8/Rrp43, and EXOSC9/Rrp45. The DIS3/Dis3/Rrp44 catalytic subunit is located at the bottom. Missense mutations in the gene encoding the EXOSC2 cap subunit (teal blue, labeled 2) are linked to a novel syndrome termed SHRF (short stature, hearing loss, retinitis pigmentosa and distinctive facies) (Di Donato et al. 2016). In contrast, missense mutations in the gene encoding the EXOSC3 cap subunit (dark blue, labeled 3 ) cause PCH1b (pontocerebellar hypoplasia type 1b) (Wan et al. 2012; Biancheri et al. 2013; Eggens et al. 2014; Halevy et al. 2014; Schottmann et al. 2017). (B) The structure and organization of the RNA exosome is highly conserved across eukaryotes. A structural model of the human nuclear RNA exosome (left) [PDB 6D6R] (Weick et al. 2018) and the S. cerevisiae nuclear RNA exosome (right) [PDB 6FSZ] (Schuller et al. 2018) are depicted with the cap subunits EXOSC1/CsI4 (Human/S. cerevisiae), EXOSC2/Rrp4, EXOSC3/Rrp40, and catalytic subunit DIS3/Dis3/Rrp44 labeled. $(C, D)$ Domain structures are shown for $(C)$ EXOSC2/Rrp4 and (D) EXOSC3/Rrp40. Each of these cap subunits is composed of three different domains: an amino-terminal domain, a putative RNA binding S1 domain, and a carboxy-terminal putative RNA binding KH (K homology) domain. The "GxNG" motif identified in the KH domain of both cap subunits is boxed in green. The position of the disease-linked amino acid substitutions in human EXOSC2 and EXOSC3 are depicted above the domain structures in red. Sequence alignments of EXOSC2/Rrp4 and EXOSC3/Rrp40 orthologs from Homo sapiens ( $\mathrm{Hs})$, Mus musculus $(\mathrm{Mm})$, and S. cerevisiae $(\mathrm{Sc})$ below the domain structures show the highly conserved residues altered in disease in red and the conserved sequences flanking these residues in gray. The amino acid substitutions in S. cerevisiae Rrp4 generated in this study and those in S. cerevisiae Rrp40, described previously (Fasken et al. 2017; Gillespie et al. 2017), that correspond to the diseaselinked amino acid substitutions in human EXOSC2 and EXOSC3, are shown below the structures in red. 
Wasmuth et al. 2017; Schuller et al. 2018; Weick et al. 2018). Mtr4/MTR4/MTREX aids the RNA exosome in targeting and processing target RNA, such as the $5.8 \mathrm{~S}$ rRNA precursor (7S rRNA), and can act as part of the budding yeast TRAMP (Trf4/5-Air1/2-Mtr4 Polyadenylation) complex or the mammalian NEXT (Nuclear Exosome Targeting) complex, both of which facilitate nuclear RNA surveillance and quality control of ncRNA by the RNA exosome (de la Cruz et al. 1998; LaCava et al. 2005; Vaňáčová et al. 2005; Houseley and Tollervey 2008, 2009; Lubas et al. 2011; Stuparevic et al. 2013; Schuch et al. 2014; Rodríguez-Galán et al. 2015; Kilchert et al. 2016b; Falk et al. 2017; Zinder and Lima 2017). Thus, interactions with these nuclear cofactors influence the function of the RNA exosome in vivo.

Recent studies have linked missense mutations in EXOSC genes encoding the structural subunits of the RNA exosome to various human pathologies, which comprise a growing family of diseases termed "RNA exosomopathies" (Wan et al. 2012; Biancheri et al. 2013; Boczonadi et al. 2014; Eggens et al. 2014; Di Donato et al. 2016; Schottmann et al. 2017; Burns et al. 2018; Fasken et al. 2020; Slavotinek et al. 2020). Intriguingly, these single amino acid substitutions often occur in highly conserved domains of the RNA exosome subunits. Mutations in the cap subunit gene EXOSC3 and the core subunit gene EXOSC8 cause forms of pontocerebellar hypoplasia (PCH1b and PCH1c, respectively), neurological disorders characterized by atrophy of the pons and cerebellum (Wan et al. 2012; Biancheri et al. 2013; Boczonadi et al. 2014; Eggens et al. 2014; Schottmann et al. 2017; Morton et al. 2018), while mutations in the core subunit genes EXOSC5 and EXOSC9 have been linked to similar neurological defects including cerebellar degeneration, neuronopathy and neurodevelopmental delays (Burns et al. 2017, 2018; Slavotinek et al. 2020). In contrast to the other exosomopathy mutations described thus far, missense mutations in the cap subunit gene EXOSC2 have been linked to a novel, complex syndrome characterized by retinitis pigmentosa, progressive hearing loss, premature aging, short stature, mild intellectual disability and distinctive gestalt (Di Donato et al. 2016), later named SHRF (short stature, hearing loss, retinitis pigmentosa and distinctive facies) (OMIM \#617763) (Yang et al. 2019). While SHRF patients do show some cerebellar atrophy (Di Donato et al. 2016), the disease phenotype is distinct from $\mathrm{PCH}$ as well as the other neurological deficits observed in patients with other exosomopathies, suggesting a unique molecular pathology linked to EXSOC2 mutations.

Whole exome sequencing of the three identified SHRF patients, representing two related patients and one unrelated patient, identified missense mutations in the EXOSC2 gene that alter conserved amino acids in this cap subunit, shown in Figure 1C (Di Donato et al. 2016).
The two related patients have a homozygous missense mutation EXOSC2 p.Gly30Val (G30V) in the amino-terminal domain of EXOSC2 (Di Donato et al. 2016). The other patient carries compound heterozygous missense mutations EXOSC2 p.Gly30Val and EXOSC2 p.Gly198Asp (G30V/G198D), with the G198D missense mutation located within the K-homology $(\mathrm{KH})$ RNA binding domain (Di Donato et al. 2016; Yang et al. 2019). These amino acid substitutions occur in highly conserved residues of EXOSC2, which are conserved across EXOSC2/Rrp4 orthologs from different eukaryotic species and conserved between EXOSC2 and the EXOSC3/Rrp40 cap subunits of the eukaryotic RNA exosome (Fig. 1D; Supplemental Fig. S1). Notably, EXOSC2 Gly30 and EXOSC3 Gly31, an amino acid that is substituted in PCH1b patients (Wan et al. 2012), are conserved and in the same position in the two cap subunits, falling within a conserved "VxPG" consensus sequence (Supplemental Fig. S1). EXOSC2 Gly198 and EXOSC3 Trp238, another amino acid that is substituted in PCH1b patients (Wan et al. 2012), lie in the KH domains of the two cap subunits, falling within or adjacent to a conserved "GxNG" motif (Fig. 1D). The "GxNG" motif is unique to the $\mathrm{KH}$ domain of these RNA exosome cap subunits and is predicted to play a structural role (Oddone et al. 2007). However, when EXOSC2 Gly30, EXOSC2 Gly198, and EXOSC3 Gly31, EXOSC3 Trp238 are substituted in compound heterozygous variants, they give rise to distinct disease phenotypes, suggesting that similar missense mutations in EXOSC2 and EXOSC3 have different mechanistic effects on the function of the RNA exosome in vivo. Therefore, to better understand the molecular pathology of these exosomopathies, including SHRF, it is necessary to investigate the molecular and functional consequences of pathogenic amino acid substitutions that underlie each disease.

A previous study provided some important insights into how the EXOSC2 mutations that cause SHRF could contribute to pathology (Yang et al. 2019). This study used several different approaches, including using patient Blymphoblasts, in vitro cell culture and a Drosophila melanogaster model depleted for the fly EXOSC2/Rrp4 ortholog. Taken together, results from this study suggest that EXOSC2 dysfunction could compromise downstream molecular pathways, including neurodevelopment and autophagy (Yang et al. 2019). While informative in probing the molecular pathology that may underlie the SHRF syndrome, a limitation of this study is that the authors did not examine known targets of the RNA exosome nor did they examine the in vivo consequences of the SHRF-linked EXOSC2 variants within a whole organism. Given that this diverse class of RNA exosomopathies arises from amino acid substitutions in structural subunits of a singular complex, assessing defects in RNA exosome function in vivo is critical for a holistic understanding of the molecular and functional consequences underlying each disease 
phenotype. Previous studies have assessed the functional and molecular consequences of exosomopathy-linked EXOSC 3 and EXOSC5 mutations in vivo using yeast and fly genetic model systems (Fasken et al. 2017; Gillespie et al. 2017; de Amorim 2020; Morton et al. 2020; Slavotinek et al. 2020). Utilizing a genetic model system to explore the consequences of the specific amino acid changes that occur in SHRF can provide insight into how RNA exosome function is altered in disease.

To explore the functional consequences of the amino acid substitutions in EXOSC2 that occur in SHRF, we took advantage of the budding yeast model system. We generated variants of the $S$. cerevisiae EXOSC2 ortholog, Rrp4, that model the pathogenic amino acid substitutions and examined their function in budding yeast. Our results show that the yeast Rrp4 G58V variant, corresponding to the EXOSC2 G30V variant, is not able to replace the function of the essential RRP4 gene. In contrast, cells that express the Rrp4 G226D variant, corresponding to the EXOSC2 G198D variant, show impaired cell growth and defects in RNA exosome function, revealing that this
Rrp4 G226D variant is functional but impaired. Based on RNA-seq analysis, the rrp4-G226D cells show broad transcriptomic changes with defects particularly in nuclear surveillance by the RNA exosome. Genetic and biochemical studies demonstrate that the rrp4-G226D variant exhibits impaired interactions with RNA exosome cofactor mutants, likely suggesting defects in association with Mtr4. Combined, these results suggest that amino acid changes in Rrp4 that model those in EXOSC2 impair the overall function of the RNA exosome, potentially through impairment of interactions with the exosome cofactor Mtr4.

\section{RESULTS}

\section{EXOSC2 amino acid substitutions linked to SHRF are located in conserved domains}

To explore how EXOSC2 G30V and EXOSC2 G198D variants could alter the structure of the EXOSC2 protein or the RNA exosome complex (Fig. 1C), we modeled these EXOSC2 amino acid substitutions using recent structures

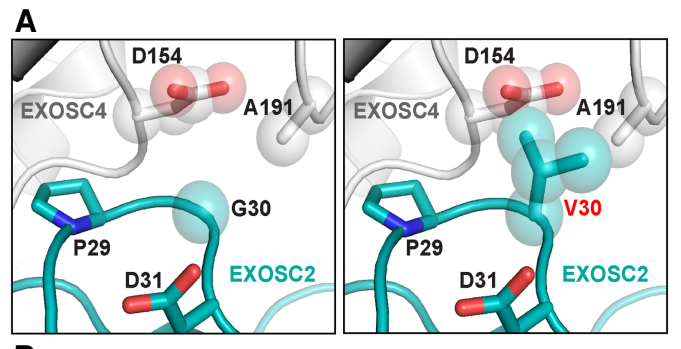

B

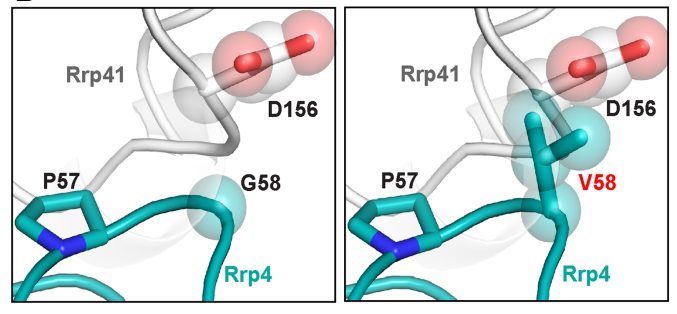

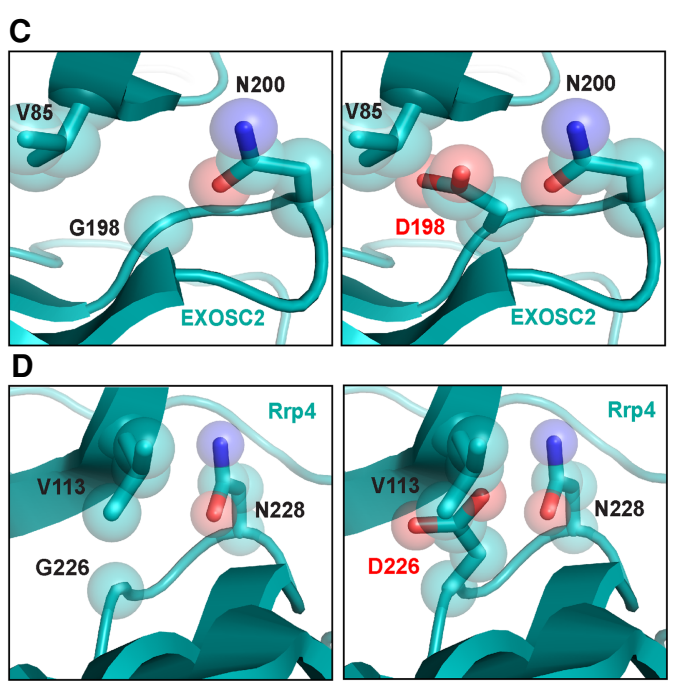

FIGURE 2. Modeling pathogenic amino acid substitutions in Human EXOSC2 and S. cerevisiae Rrp4. (A) Structural modeling of the human EXOSC2 p.Gly30Val (G30V) amino acid substitution identified in patients with SHRF syndrome in the human RNA exosome. Zoomed-in representations of the interface between EXOSC2 (teal blue) and EXOSC4 (light gray) modeling the native EXOSC2 Gly30 (G30) residue (left) or the pathogenic EXOSC2 Val30 (V30) residue (right) are depicted. The EXOSC2 Gly30 residue is located in the amino-terminal domain of EXOSC2, near the interface of EXOSC2 with the core subunit, EXOSC4. (B) Structural modeling of budding yeast Rrp4 Gly58Val (G58V) amino acid change, corresponding to EXOSC2 p.Gly30Val amino acid change, in the budding yeast exosome. Zoomed-in representations of the interface between Rrp4 (teal blue) and the budding yeast EXOSC4 ortholog, Rrp41 (light gray), modeling the native Rrp4 Gly58 (G58) residue (left) or the modeled pathogenic Rrp4 Val58 (V58) residue (right) are shown. The Rrp4 Gly58 residue is conserved between human and yeast and, similarly to EXOSC2 Gly30, is located in the amino-terminal domain of Rrp4, near the interface of Rrp4 with the core subunit, Rrp41. (C) Structural modeling of the EXOSC2 p.Gly198Asp (G198D) amino acid substitution identified in patients with SHRF syndrome in the human RNA exosome. Zoomed-in representations of EXOSC2 modeling the native EXOSC2 Gly198 (G198) residue (left) or the pathogenic EXOSC2 Asp198 (D198) residue (right) are shown. The EXOSC2 Gly198 residue is located in the KH-domain of EXOSC2 within a dense region of the protein, surrounded by four $\beta$-sheets. (D) Structural modeling of the budding yeast Rrp4 Gly226Asp (G226D) amino acid change, corresponding to the EXOSC2 p.Gly198Asp amino acid change, in the budding yeast RNA exosome. Zoomed-in representations of Rrp4 modeling the native Rrp4 Gly226 (G226) residue (left) or the modeled pathogenic Rrp4 Asp226 (D226) residue (right) are shown. The Rrp4 Gly226 residue, which is conserved between human and yeast, is located in the $\mathrm{KH}$-domain of Rrp4 within a dense region of the protein, surrounded by four $\beta$-sheets. Structural modeling in $A$ and $C$ was performed with the human RNA exosome structure (PDB 6D6R) (Weick et al. 2018) and in B and D with the yeast RNA exosome structure (PDB 6FSZ) (Schuller et al. 2018) using PyMOL (The PyMOL Molecular Graphics System, Version 2.0 Schrödinger, LLC). 
of the human RNA exosome (PDB 6D6R [Fig. 2A,C; Weick et al. 2018]) and the S. cerevisiae RNA exosome (PDB 6FSZ [Fig. 2B,D; Schuller et al. 2018]). Structural modeling shows that the EXOSC2 Gly30 residue is positioned at the interface with the core subunit EXOSC4 toward the exterior of the complex in a region with little disorder (Fig. 2A). The EXOSC2 Gly30 residue lies in a $\beta$-turn next to a highly conserved proline (Pro29), which is likely essential for the region to have the flexibility needed to make the sharp turn observed in the structure. EXOSC2 Gly30 is also adjacent to an aspartic acid (Asp31), which forms a salt bridge with Arg232 of EXOSC4, likely stabilizing the interaction between the two subunits (Supplemental Fig. S2A). An amino acid substitution that alters the glycine at position 30 is predicted to alter the $\beta$-turn and position the Asp31 residue away from Arg232 such that the salt bridge would be disrupted. In addition, the EXOSC2 G30V substitution introduces a valine, which is significantly larger than glycine and appears to clash with residues Asp154 and Ala191 in EXOSC4 (Fig. 2A), suggesting this substitution could negatively impact the interactions between the cap EXOSC2 and core EXOSC4 subunit. In the budding yeast structure, Rrp4 Gly58, corresponding to EXOSC2 Gly30 (Fig. 1C), is located at the interface with Rrp41, the budding yeast EXOSC4 ortholog, in a $\beta$ turn adjacent to a highly conserved proline (Pro57) in Rrp4 (Fig. 2B), mirroring the human structural model (Fig. 2A). Rrp4 Gly58 is located next to a glutamic acid (Glu59; Supplemental Fig. S2B) in Rrp4 that forms a salt bridge with Arg233 in Rrp41, similar to the EXSOC2EXOSC4 interface (Supplemental Fig. S2A). Similar to the human structure, in the yeast exosome structure, the Rrp4 Gly58 residue is also predicted to be essential for the flexibility of the region, facilitating the $\beta$-turn, and thus stabilizing the Rrp4-Rrp41 interface (Supplemental Fig. S2B). Similar to the EXOSC2 G30V substitution, substitution of Gly58 in Rrp4 most likely disrupts this $\beta$-turn, disrupting the salt bridge and destabilizing the Rrp4-Rrp41 interface.

EXOSC2 Gly198 is positioned in a dense region of the subunit, surrounded by four $\beta$ sheets (Fig. 2C). The EXOSC2 G198D substitution introduces the large aspartic acid residue which appears to clash with neighboring residues Val85 and Asn200 (Fig. 2C) and alter EXOSC2 conformation. In addition, the EXOSC2 G198D substitution introduces a polar aspartic acid residue in place of glycine with an electronegative oxygen that would undergo repulsion with the oxygen of Asn200, making the native structure depicted in Figure 2C unlikely for this variant. Structural modeling of the Rrp4 Gly226 residue, corresponding to EXOSC2 Gly198 (Fig. 1C), shows that, like Gly198 in EXOSC2, Gly226 residue is positioned in a dense region of Rrp4, surrounded by four $\beta$ sheets (Fig. 2D). The residues neighboring Rrp4 Gly226, Val113, and Asn228, are highly conserved and correspond to
EXOSC2 Val85 and Asn200, suggesting that the budding yeast Rrp4 G226D substitution can accurately model the structural changes predicted for the human EXOSC2 G198D substitution.

The online server mCSM-PPI2 was used to calculate the change in Gibbs free energy $(\Delta \Delta G)$ to predict the effect of the EXOSC2 amino acid substitutions and corresponding Rrp4 amino acid substitutions on protein-protein interactions. Consistent with observations from structural modeling, the software predicts destabilizing changes in the affinity of the protein-protein interactions for both EXOSC2 G30V $(\Delta \Delta \mathrm{G}=-1.012 \mathrm{Kcal} / \mathrm{mol})$ and EXOSC2 G198D $(\Delta \Delta \mathrm{G}=-0.509 \mathrm{Kcal} / \mathrm{mol})$. The EXOSC2 G198D substitution is also predicted to reduce protein stability (Score $=1.000$ Polymorphism Phenotyping v2). These predictions are consistent with previous work showing that EXOSC2 G198D has reduced stability compared to wildtype EXOSC2 (Yang et al. 2019). Furthermore, both substitutions are strongly predicted to have deleterious effects on EXOSC2 function (G30V score -7.938 and G198D score -6.35 calculated by PROVEAN; G30V score 91, G198D score 94 calculated by SNAP-2). Both Rrp4 G58V (which models EXOSC2 G30V) and Rrp4 G226D (which models EXOSC2 G198D) are predicted to decrease protein stability (Score $=1.000$ Polymorphism Phenotyping v2) as well as to have deleterious effects on function (G58V score -8.981 and G226D score -6.517 calculated by PROVEAN). Rrp4 G58V is likely to alter the native protein (score 63 calculated by SNAP2), though to a slightly lower degree than calculated for the human EXOSC2 G30V variant. However, Rrp4 G226D likely results in a change to the native protein (score 92 by SNAP2), mirroring the strong effect predicted for the human EXOSC2 G198D variant. In conclusion, these in silico predictions (summarized in Supplemental Table S3) suggest that the pathogenic amino acid substitutions have molecular consequences that could affect RNA exosome function in both humans and budding yeast.

\section{Saccharomyces cerevisiae Rrp4 variants that model the pathogenic EXOSC2 variants impair RNA exosome function}

To assess the in vivo consequences of the pathogenic amino acid substitutions in EXOSC2, G30V, and G198D, we generated the corresponding amino acid changes in the S. cerevisiae ortholog Rrp4, G58V, and G226D (Fig. 1C). As all core RNA exosome subunits genes are essential in budding yeast (Allmang et al. 1999), we first assessed whether these rrp4 gene mutants can replace the essential RRP4 gene. To facilitate comparison of different rrp4 mutants, we used a plasmid shuffle assay in which cells deleted for the genomic copy of RRP4 are transformed with plasmids containing mutant alleles (See Materials and Methods). This approach ensures that the genetic 
background for all mutants compared to one another is identical (Sikorski and Hieter 1989). In this plasmid shuffle assay, rrp $4 \Delta$ cells containing a RRP4 maintenance plasmid and either rrp4-G58V or rrp4-G226D plasmid were serially diluted and spotted onto 5-FOA plates to select for cells that harbor the rrp4 mutant as the sole copy of RRP4 (Fig. 3A). The rrp4-G58V mutant cells are not viable at any temperature tested, whereas the rrp4-G226D cells exhibit impaired growth at $37^{\circ} \mathrm{C}$ as compared to control
RRP4 cells (Fig. 3A). Control cells expressing wild-type RRP4 grow at all temperatures as expected. The impaired growth of rrp4-G226D mutant cells was further analyzed by serial dilution and spotting on solid minimal media (Fig. 3B) and in a liquid media growth assay (Fig. 3C). On solid media and in liquid culture, the rrp4-G226D cells show impaired growth at $37^{\circ} \mathrm{C}$ compared to control RRP4 cells (Fig. 3B,C). For comparison, we also assessed the growth of the previously characterized rrp40-W195R mutant cells
A

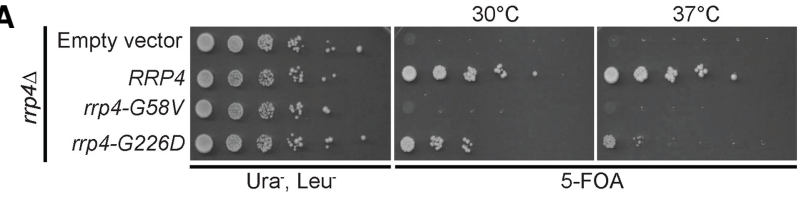

B

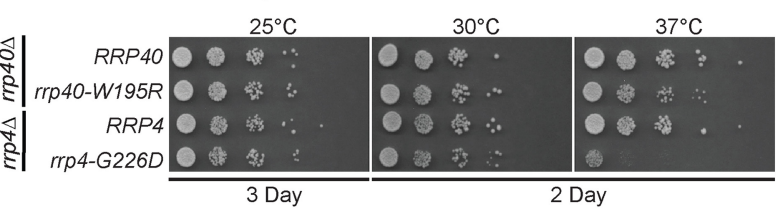

D

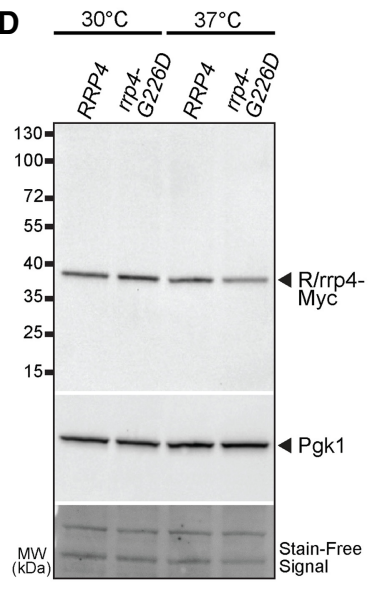

E

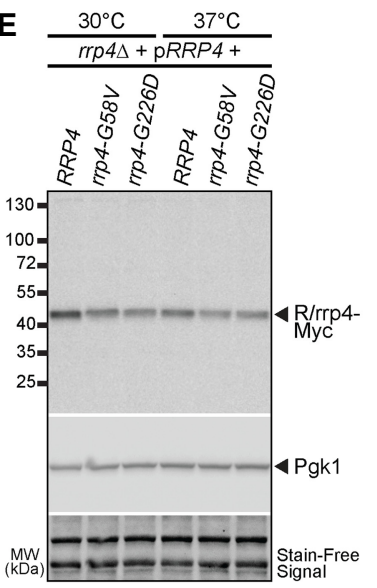

C

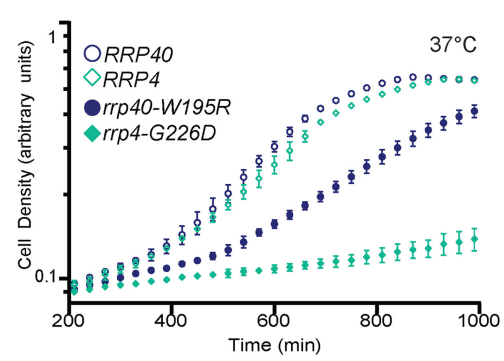

$\mathbf{F}$

G
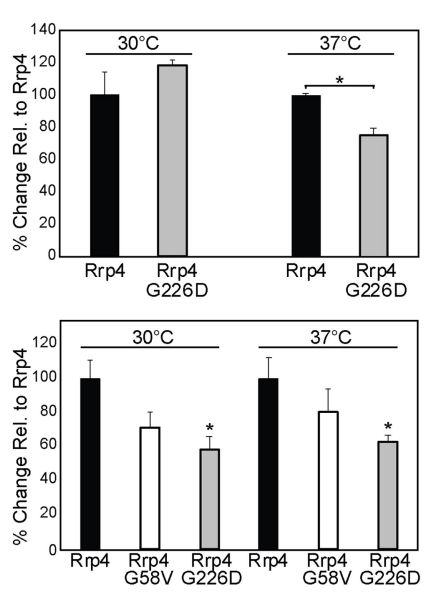

FIGURE 3. S. cerevisiae Rrp4 variants that model EXOSC2 variants identified in patients show impaired function. S. cerevisiae cell expressing Rrp4 variants that model pathogenic amino acid changes found in EXOSC2 were generated as described in Materials and Methods. ( $A$ ) Although cell growth is comparable for all mutants that contain a wild-type RRP4 maintenance plasmid (Ura ${ }^{-}$Leu $^{-}$), rrp4-G58V mutant cells are not viable on plates containing 5-FOA where the maintenance plasmid is not present. The rrp4-G226D cells show temperature-sensitive growth on 5-FOA relative to control RRP4 cells. The cells were grown at the indicated temperatures. $(B, C)$ The rrp4-G226D cells exhibit profoundly impaired growth compared to control $R R P 4$ cells at $37^{\circ} \mathrm{C}$ as assessed by $(B)$ serial dilution growth assay on plates or $(C)$ growth in liquid media. $(B)$ The rrp $4 \Delta$ cells expressing only RRP4 or rrp4-G226D and rrp40 $\Delta$ cells expressing only RRP40 or rrp40-W195R were serially diluted, spotted onto solid media grown at the indicated temperatures or $(C)$ grown in liquid media at $37^{\circ} \mathrm{C}$ with optical density measurement used to assess cell density over time. The growth of rrp40-W195R cells, previously reported to be moderately impaired at $37^{\circ} \mathrm{C}$ (Fasken et al. 2017; Gillespie et al. 2017), was included as a comparative control. (D) The steady-state level of the Rrp4 G226D protein variant is modestly decreased at $37^{\circ} \mathrm{C}$. Lysates of $r r p 4 \Delta$ cells solely expressing Myc-tagged wild-type Rrp4 or rrp4-G226D grown at $30^{\circ} \mathrm{C}$ or $37^{\circ} \mathrm{C}$ were analyzed by immunoblotting with an anti-Myc antibody to detect Rrp4-Myc and an anti-Pgk1 antibody to detect 3-phosphoglycerate kinase (Pgk1) as a loading control. (E) The Rrp4-G58V protein variant is expressed and the steady-state level of the Rrp4 G226D protein variant is decreased in cells coexpressing wild-type Rrp4. Lysates of $\operatorname{rrp} 4 \Delta$ cells coexpressing untagged wild-type Rrp4 and Myc-tagged wild-type Rrp4, Rrp4 G58V, or Rrp4 G226D grown at 30 ${ }^{\circ} \mathrm{C}$ were analyzed by immunoblotting with an anti-Myc antibody to detect Rrp4-Myc and anti-Pgk1 antibody to detect 3-phosphoglycerate kinase (Pgk1) as loading control. (F) Quantitation of the percentage of Rrp4 or Rrp4 G226D protein detected in lysates of rrp4 $\Delta$ cells solely expressing Myc-tagged Rrp4 or Rrp4 G226D grown at $30^{\circ} \mathrm{C}$ or $37^{\circ} \mathrm{C}$. Graph shows the mean percentage of Rrp4-Myc protein from three independent experiments ( $n$ $=3$ ). Error bars represent standard error of mean. Statistical significance is denoted by asterisk (* $P$-value $\leq 0.05$ ). (G) Quantitation of the percentage of Rrp4, Rrp-G58V and Rrp4-G226D protein detected in lysates of $r r p 4 \Delta$ cells expressing Myc-tagged Rrp4 or Rrp4 variants grown at $30^{\circ} \mathrm{C}$ or $37^{\circ} \mathrm{C}$. Graph shows the mean percentage of Rrp4-Myc from three independent experiments $(n=3)$. Error bars represent standard error of mean. Statistical significance is denoted by asterisk ( ${ }^{*} P$-value $\left.\leq 0.05\right)$. Quantitation of immunoblots in $F$ and $G$ was performed as described in Materials and Methods. 
(Fasken et al. 2017; Gillespie et al. 2017), which solely express the rrp40-W195R mutant corresponding to the EXOSC3-W238R mutant linked to PCH1b (Wan et al. 2012; Biancheri et al. 2013; Eggens et al. 2014; Halevy et al. 2014; Schottmann et al. 2017). The rrp4-G226D cells exhibit a more profound growth defect than rrp40-W195R cells at $37^{\circ} \mathrm{C}$ as determined by comparing each mutant to the corresponding wild-type control (Fig. 3B,C).

The growth defects associated with the rrp4 mutant cells could be due to a decrease in the level of the essential Rrp4 protein. To explore this possibility, we examined the expression of Myc-tagged wild-type Rrp4 and Rrp4 variants by immunoblotting and quantitated the changes in steady-state level of Rrp4 G226D-Myc and Rrp4 G58VMyc compared to wild-type control (Fig. 3D-F). We first examined the steady-state levels of Myc-tagged Rrp4 G226D when expressed as the sole copy of the Rrp4 protein in rrp4s cells grown at either $30^{\circ} \mathrm{C}$ or $37^{\circ} \mathrm{C}$. Immunoblotting reveals that the steady-state level of Rrp4 G226D is comparable to wild-type $\operatorname{Rrp} 4$ at $30^{\circ} \mathrm{C}$; however, at $37^{\circ} \mathrm{C}$, the level of Rrp4 G226D is decreased to $\sim 75 \%$ of that of wild-type Rrp4 (Fig. 3D). As Rrp4 G58V does not support cell viability, we could not examine the expression of this variant as the sole copy of Rrp4 in cells. Thus, we examined the expression of Myc-tagged Rrp4, Rrp4 G58V, and Rrp4 G226D in the presence of RRP4. Under these conditions, where an untagged copy of Rrp4 is present, the steady-state level of Rrp4 G58VMyc is decreased to $\sim 68 \%$ that of wild-type Rrp4 and Rrp4 G226D-Myc is decreased to $~ 51 \%$ that of the wildtype Rrp4 at both $30^{\circ} \mathrm{C}$ and $37^{\circ} \mathrm{C}$ (Fig. 3E). These data show that the level of Rrp4 G58V is not decreased more than Rrp4 G226D in the presence of a wild-type copy of Rrp4. Quantitation of results from these studies are shown in Figure 3F and G. The Rrp4 variants show a decrease in steady-state levels in the presence of wild-type Rrp4, suggesting cells can discriminate between wild-type and variant RNA exosome subunits.

\section{The Rrp4 G226D variant can associate with the RNA exosome complex in vivo in the absence of competing wild-type Rrp4}

The amino acid substitutions in the Rrp4 variants could decrease association of Rrp4 with the other cap and/or core subunits of the RNA exosome, as reported for human EXOSC2 G30V and EXOSC2 G198D (Yang et al. 2019). To initially examine the association of Rrp4 variants with the RNA exosome, we performed coimmunoprecipitations using RRP43-TAP cells that contain the endogenous RRP4 gene and express a carboxy-terminally tandem affinity purification (TAP)-tagged Rrp43 core subunit from the endogenous RRP43 locus. We coexpressed Rrp4-Myc, Rrp4 G58V-Myc, or Rrp4 G226D-Myc in these RRP43-TAP cells. The Rrp43-TAP protein was immunoprecipitated and asso- ciation of the Myc-tagged Rrp4 variants was assayed by immunoblotting (Fig. 4A). Under these conditions where an endogenous, wild-type copy of RRP4 is present, we do not detect association of Rrp4 G58V-Myc or Rrp4 G226D-Myc with Rrp43-TAP, whereas we do detect association of the wild-type Rrp4-Myc with Rrp43-TAP (Fig. 4A).

To further investigate association of Rrp4 G226D with the RNA exosome complex, we performed the same coimmunoprecipitation experiment in the absence of endogenous RRP4 using RRP43-TAP rrp4 4 cells that express Rrp4-Myc or Rrp4 G226D-Myc. We could not express Rrp4 G58V-Myc in these cells as Rrp4 G58V does not support viability (Fig. 3A). The Rrp43-TAP protein was immunoprecipitated and association of Rrp4-Myc or Rrp4 G226DMyc was assayed by immunoblotting (Fig. 4B). Under these conditions where Rrp4 G226D-Myc is the sole copy of the essential cap subunit, we detect association with the RNA exosome complex at levels comparable to wild-type Rrp4-Myc (Fig. 4B). These data suggest that Rrp4 G226D can associate with the RNA exosome complex when it is the sole copy of the cap subunit; however, in the presence of endogenous RRP4, the wild-type copy of Rrp4 can out compete pathogenic Rrp4 variants for incorporation into the RNA exosome complex.

\section{The Rrp4 G226D variant impairs RNA exosome function}

To assess the function of the RNA exosome in rrp4-G226D cells, we examined the steady-state levels of several welldefined RNA exosome target transcripts. The RNA exosome has a critical role in ribosomal RNA (rRNA) processing, specifically processing 7S pre-rRNA into mature 5.8S rRNA (Mitchell et al. 1996; Allmang et al. 1999). We analyzed the processing of 5.8S rRNA in rrp4-G226D cells using northern blotting. We also compared 5.8S rRNA processing in rrp4-G226D cells to yeast cells modeling EXOSC3 PCH1b mutations, rrp40-G8A and rrp40-W195R (Fasken et al. 2017; Gillespie et al. 2017). As shown in Figure 5A, rrp4-G226D cells accumulate 7S pre-rRNA, a precursor of mature $5.8 \mathrm{~S}$ rRNA. In addition, several intermediate precursors of $5.8 \mathrm{~S}$ rRNA, indicated by asterisks, accumulate in rrp4-G226D cells. Despite the accumulation of precursors, the level of mature 5.8S rRNA does not appear to differ in rrp4-G226D cells compared to control RRP4 cells. The accumulation of $7 \mathrm{~S}$ pre-rRNA and other 5.8S rRNA precursors in rrp4-G226D cells is greater than that detected in rrp40-W195R cells (Fig. 5A; Supplemental Fig. S3), which have documented accumulation of this rRNA precursor (Gillespie et al. 2017).

We next analyzed the steady-state levels of several RNA exosome target transcripts in rrp4-G226D cells using quantitative RT-PCR (Allmang et al. 1999). The rrp4-G226D cells exhibit a significant increase in the level of $3^{\prime}$-extended U4 pre-snRNA compared to RRP4 control 

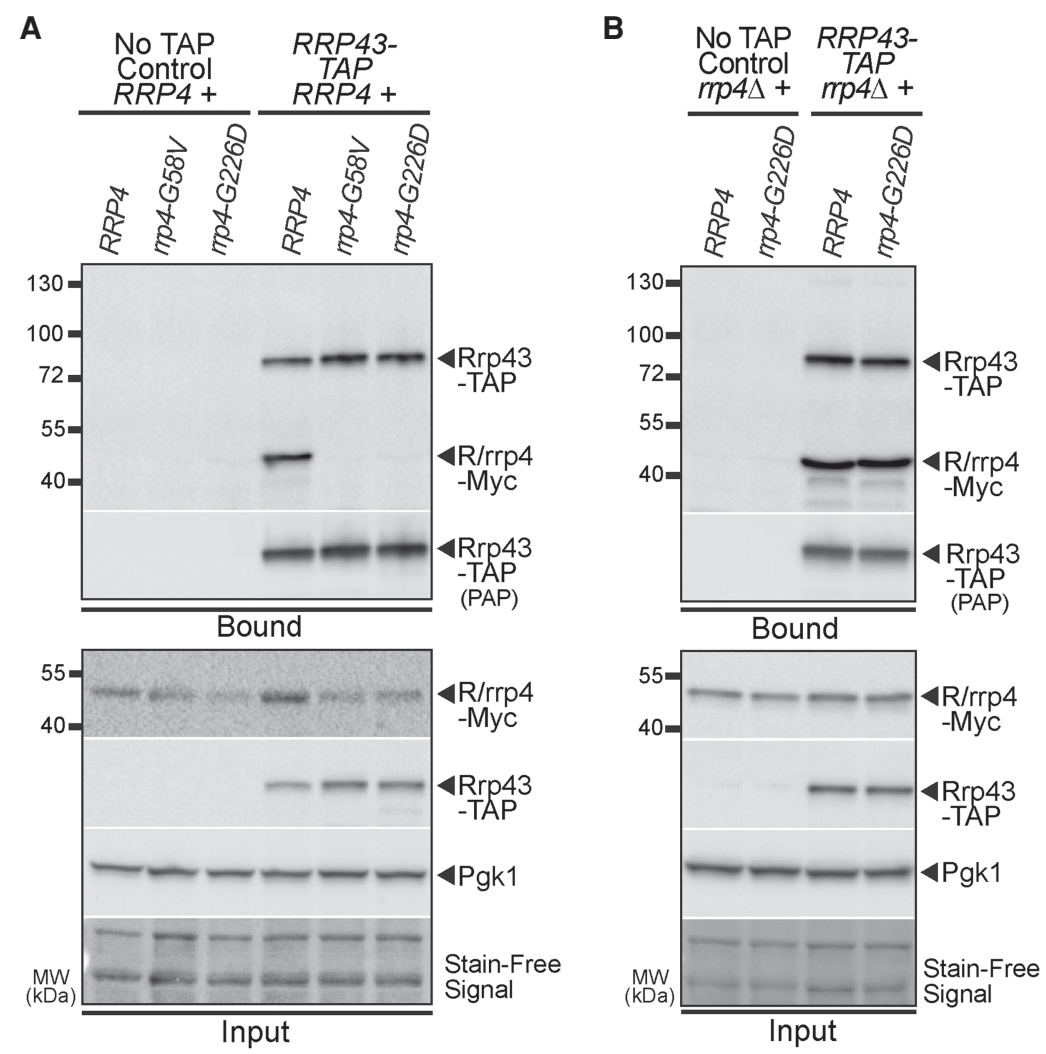

FIGURE 4. The Rrp4 G58V and Rrp4 G226D variants do not associate with the RNA exosome complex in the presence of wild-type Rrp4, but the Rrp4 G226D variant can associate with the RNA exosome complex. (A) The Rrp4 G226D and Rrp4 G58V variants do not associate with the RNA exosome core subunit Rrp43 in the presence of a wild-type copy of Rrp4. TAP-tagged Rrp43 was immunoprecipitated from RRP43-TAP cells expressing Myc-tagged Rrp4, Rrp4 $\mathrm{G} 58 \mathrm{~V}$, or Rrp4 $\mathrm{G} 226 \mathrm{D}$ in the presence of wild-type Rrp4 grown at $30^{\circ} \mathrm{C}$ using lgG Sepharose beads and analyzed by immunoblotting. As a control, immunoprecipitations were also performed from untagged RRP43 cells (No TAP Control) expressing Myc-tagged Rrp4 proteins. The bound/input level of Rrp4-Myc was detected with an anti-Myc antibody and bound/input level of Rrp43-TAP was detected with a peroxidase antiperoxidase (PAP) antibody. Bound Rrp43-TAP was also detected by the anti-Myc antibody as the Protein A moiety of the TAP tag binds to antibody. The input level of 3-phosphoglycerate kinase (Pgk1) was detected with an anti-Pgk1 antibody as a loading control. The stain-free signal from input protein is also included as a loading control. (B) The Rrp4 G226D variant associates with the RNA exosome core subunit Rrp43 at a level similar to wild-type Rrp4 when the variant is the sole form of Rrp4. TAP-tagged Rrp43 was immunoprecipitated from RRP43-TAP rrp4A cells expressing either Myc-tagged wild-type Rrp4 or Rrp4 G226D, which were grown at $30^{\circ} \mathrm{C}$ using IgG Sepharose beads and analyzed by immunoblotting. As a control, immunoprecipitations were also performed from untagged RRP43 rrp4 4 cells (No TAP Control) expressing Myctagged Rrp4 proteins. The bound/input level of Rrp4-Myc was assessed with an anti-Myc antibody and bound/input level of Rrp43-TAP was detected with a peroxidase anti-peroxidase (PAP) antibody. Bound Rrp43-TAP was also detected by the anti-Myc antibody as the Protein A moiety of the TAP tag binds to antibody. The input level of 3-phosphoglycerate kinase (Pgk1) was detected with an anti-Pgk1 antibody as a loading control. The immunoblots are representative of triplicate experiments; coimmunoprecipitations were performed as described in Materials and Methods.

cells (Fig. 5B). The rrp4-G226D cells exhibit a significant increase in the level of U14 box C/D snoRNA, whereas they show no significant difference in the level of the snR44 box H/ACA snoRNA (Fig. 5C). We also measured the steadystate level of the telomerase RNA TLC1, which is pro- cessed by the RNA exosome in a pathway similar to pre-snRNA processing (Coy et al. 2013). The rrp4G226D cells exhibit a significant increase in the steady-state level of both the mature and the extended precursor form of TLC1 compared to RRP4 cells (Fig. 5D). These data indicate that known RNA exosome target transcripts accumulate in rrp4-G226D cells and suggest that Rrp4 G226D impairs the function of the RNA exosome.

\section{The Rrp4 G226D variant causes broad transcriptomic changes}

To further investigate the molecular consequences of the Rrp4 G226D substitution, we performed RNA-seq analysis on rRNA-depleted total RNA isolated from three independent biological replicates of the rrp4-G226D and control RRP4 cells as described in Materials and Methods. Unbiased principal component analysis (PCA) of the resulting RNA-seq data produced two distinct clusters, indicating that the rrp4 mutant transcriptome is distinct from the wild-type RRP4 control (Fig. 6A). This separation between the two genotypes and reproducibility among the RNA-seq replicates allowed us to identify transcriptomic changes in rrp4-G226D mutant cells compared to the control (Fig. 6B). From differential gene expression analysis, we detect 860 transcripts increased $(\geq+1.5$ Fold Change $[F C]$, $P<0.05)$ and 802 transcripts decreased $(\geq-1.5 F C, P<0.05)$ in rrp4G226D cells compared to the RRP4 control (Fig. 6B). Of the 860 transcripts increased, only a third are mRNAs (34\%, 296 transcripts), with the majority being cryptic unstable transcripts (CUTs), stable unannotated transcripts (SUTs), and other ncRNAs (Fig. 6C). Consistent with the role the RNA exosome plays in degradation of nascent ncRNA species, the CUTs and SUTs combined make up the majority (65\%) of transcripts that show a steady-state increase in rrp4-G226D cells (Fig. 6C). Of the 802 transcripts decreased, the majority are mRNAs $(90 \%$, 719 transcripts) (Fig. 6C), with the most significantly 

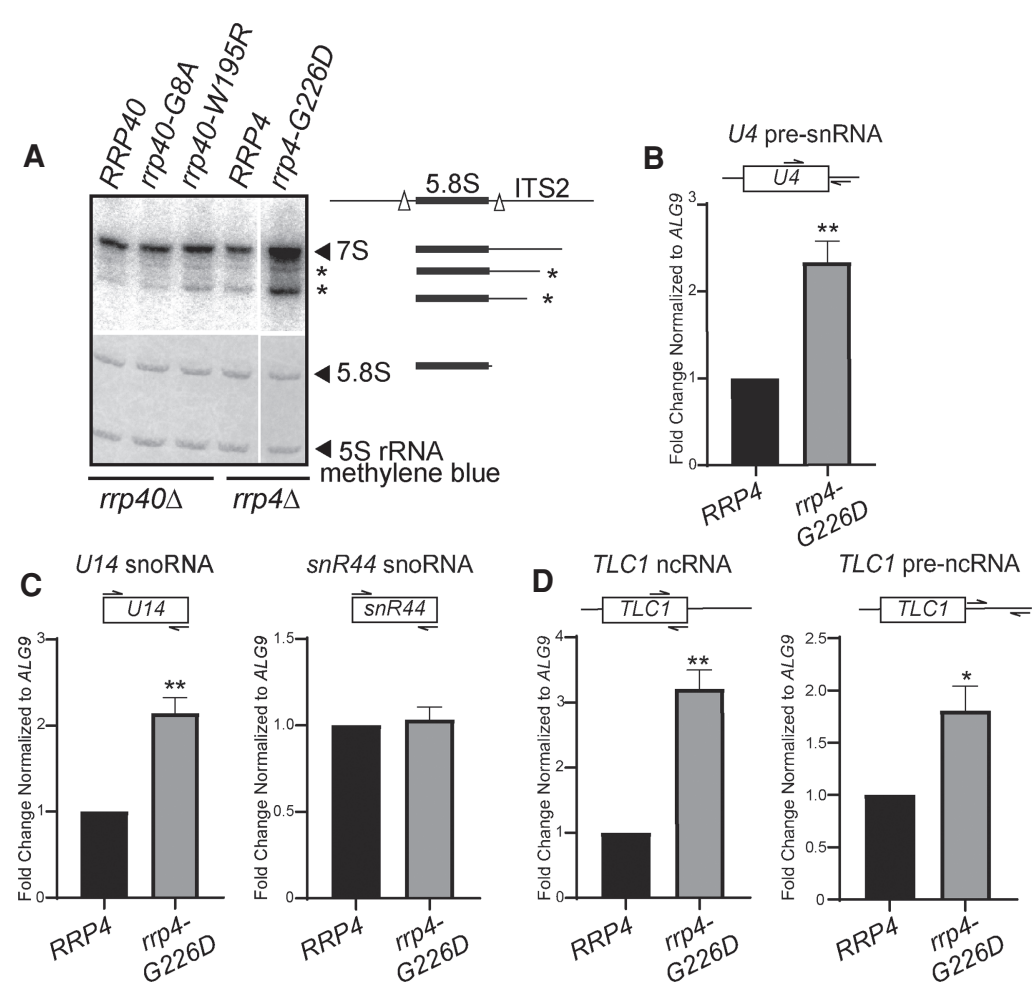

FIGURE 5. The rrp4-G226D variant cells show elevated levels of some but not all RNA exosome target transcripts. (A) The rrp4-G226D cells exhibit greater accumulation of 7S preRNA compared to RRP4 and rrp40-W195R cells grown at $37^{\circ} \mathrm{C}$. Total RNA from RRP40, rrp40-G8A, rrp40-W195R, RRP4, and rrp4-G226D cells grown at $37^{\circ} \mathrm{C}$ was analyzed by northern blotting with a 5.8S-ITS2 probe to detect 7S pre-rRNA. Mature 5.8S rRNA and 5S rRNA were detected by methylene blue staining as a loading control. The 7S pre-rRNA is normally processed to mature $5.8 \mathrm{~S}$ rRNA by $3^{\prime}-5^{\prime}$ decay of the internal transcribed spacer 2 (ITS2) via the nuclear RNA exosome (Mitchell et al. 1996; Allmang et al. 1999). All lanes are imaged from the same northern blot with a gap in the loading indicated by the white line. The simplified schematics to the right illustrate the processing steps of 7S rRNA precursor following endonucleolytic cleavage from the larger $27 \mathrm{~S}$ precursor (indicated by white triangles). (B) The rrp4-G226D cells show an elevated steady-state level of 3'-extended pre-U4 snRNA relative to RRP4 cells at $37^{\circ} \mathrm{C}$. (C) The rrp4-G226D cells exhibit an increased steady-state level of $U 14$ (snR128) snoRNA but not snR44 snoRNA relative to RRP4 cells at $37^{\circ} \mathrm{C}$. (D) The rrp4G226D cells show an elevated steady-state level of mature and extended precursor TLC1 telomerase component ncRNA relative to RRP4 cells at $37^{\circ} \mathrm{C}$. In $B-D$, total RNA was isolated from cells grown at $37^{\circ} \mathrm{C}$ and transcript levels were measured by RT-qPCR using gene specific primers, normalized relative to RRP4, and graphed as described in Materials and Methods. Gene specific primer sequences are summarized in Supplemental Table S2 and their locations within the transcript are graphically represented by the cartoons above each bar graph. Within the cartoon transcript, the box represents the body of the mature transcript. Error bars represent standard error of the mean from three biological replicates. Statistical significance of the RNA levels in rrp4-G226D cells relative to RRP4 cells is denoted by an asterisk ( ${ }^{*} P$-value $\leq 0.05 ;{ }^{* *} P$-value $\left.\leq 0.01\right)$.

decreased transcript ( $\geq-4 \mathrm{FC}$ ) being INO1, an mRNA that encodes inositol-3-phosphate synthetase (Donahue and Henry 1981; Klig and Henry 1984), which has previously been identified as a transcript bound to the catalytic subunit of the RNA exosome (Delan-Forino et al. 2017).

Gene Ontology (GO) analysis of the differentially expressed transcripts in rrp4-G226D cells using YeastEnrichr (Chen et al. 2013; Kuleshov et al. 2016, 2019) reveals that the ncRNA catabolic process is the most significant biological process category for the increased transcripts (Combined score 19.56) and cytoplasmic translation is the most significant category for the decreased transcripts (Combined score 600.4) (Fig. 6D). These $\mathrm{GO}$ analyses align with the transcripts that are altered, as two significantly decreased mRNAs ( $\geq-1.5 \mathrm{FC}$ ), RPS3 and RPL15A, encode components of the ribosome, and two significantly increased mRNAs $(\geq+1.5 \mathrm{FC})$, NRD1 and NAB3, encode components of the Nrd1-Nab3-Sen1(NNS) transcription termination complex (Steinmetz and Brow 1998; Steinmetz et al. 2001; Wolin et al. 2012; Belair et al. 2018).

To validate altered gene expression detected in the RNA-seq analysis, we measured the levels of a subset of transcripts (Fig. 7). We performed this analysis on select coding and noncoding transcripts (labeled in Fig. $6 \mathrm{~B})$. This analysis confirms that the steady-state levels of three noncoding CUTs-CUT501, CUT770, CUT896 (Fig. 7A) - and three mRNAs -PTH4, NRD1, NAB3 (Fig. 7C,D)— that increased in the RNA-seq analysis are significantly increased $(P<0.05)$ in rrp4-G226D cells compared to RRP4 control cells. We also validated decreased steady-state levels of several mRNAs (RPS3, RPL15A, INO1, HXK2, TDH1) $(P<0.01)$ in rrp4-G226D cells compared to control (Fig. 7B,C).

To compare the molecular consequences resulting from two pathogenic missense mutations in RNA exosome cap subunits, EXOSC2/ Rrp4 and EXOSC3/Rrp40 (Fasken et al. 2017; Gillespie et al. 2017), we expanded the RT-qPCR analysis to include rrp40-W195R cells. Intriguingly, we found that some altered targets in rrp4-G226D cells are affected in both mutants, while others are significantly affected only in the rrp4 mutant. The steady-state levels of CUT501, CUT770, and CUT896 are only significantly increased in rrp4G226D cells and not in rrp40-W195R cells (Fig. 7A). Steady-state levels of coding RSP3, RPL15A, and INO1 mRNAs are significantly decreased in both rrp-G226D and rrp40-W195R cells compared to control cells (Fig. 7B). In contrast, the decrease in steady-state levels of HXK2 and TDH1 mRNAs is unique to the rrp4-G226D cells, 
A

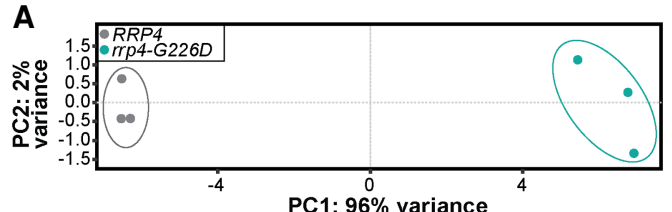

B

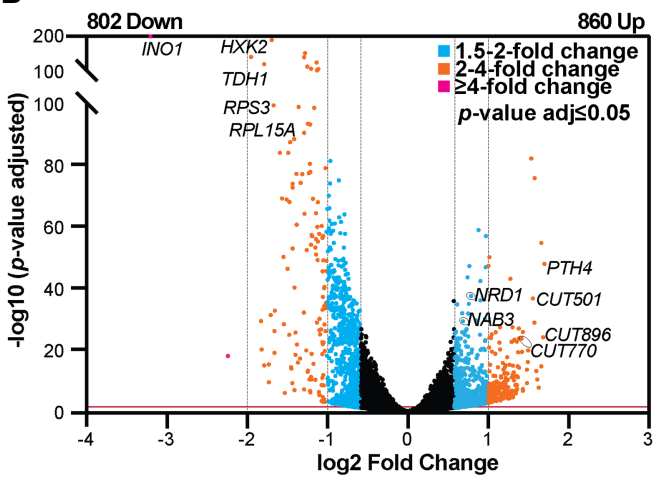

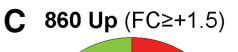

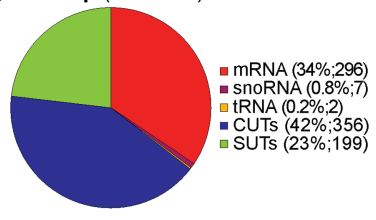

802 Down (FC $\geq-1.5)$

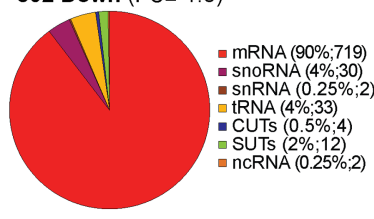

Combined Scores

D Up Genes: Go Biological Process

\begin{tabular}{|lr|}
\hline antisense RNA transcript catabolic process (GO:0071041) & 19.56 \\
ncRNA catabolic process (GO:0034661) & 16.39 \\
endonucleolytic cleavage in 5'-ETS of tricistronic rRNA transcript (GO:0000480) & 15.33 \\
SnoRNA 3'-end processing (GO:0031126) & 13.99 \\
\hline endonucleolytic cleavage to generate mature 5'-end of SSU-rRNA (GO:0000472) & 13.74 \\
\hline oligopeptide transport (GO:0006857) & 13.22 \\
anaphase-promoting complex-dependent catabolic process (GO:0031145) & 13.19 \\
rRNA 5'-end processing (GO:0000967) & 13.05 \\
\hline endonucleolytic cleavage of tricistronic rRNA transcript (GO:0000479) & 12.85 \\
\hline sister chromatid segregation (GO:0000819) & 12.58 \\
\hline Down Genes: GO Biological Process & Combined Scores \\
\hline cytoplasmic translation (GO:0002181) & 600.4 \\
translation (GO:0006412) & 510.49 \\
ribosome assembly (GO:0042255) & 119.63 \\
nicotinamide nucleotide metabolic process (GO:0046496) & 84.86 \\
peptide biosynthetic process (GO:0043043) & 80.11 \\
cellular macromolecule biosynthetic process (GO:0034645) & 79.46 \\
ribosomal small subunit assembly (GO:0000028) & 78.84 \\
pyruvate metabolic process (GO:0006090) & 77.66 \\
ribonucleoprotein complex assembly (GO:0022618) & 76.01 \\
\hline glycolytic process (GO:0006096) & 73.95 \\
\hline
\end{tabular}

FIGURE 6. RNA-seq analysis of rrp4-G226D cells reveal distinct transcriptomic changes compared to RRP4 cells. (A) Principal component analysis (PCA) of RNA-seq data collected from triplicate RRP4 and rrp4-G226D cell samples shows that the gene expression patterns from independent rrp4-G226D samples are similar and thus cluster together, but are distinct from RRP4 samples, which also cluster together. (B) A volcano plot of the differentially expressed transcripts in rrp4-G226D cells compared to RRP4 cells shows that 860 transcripts are significantly Up and 802 transcripts are Down by 1.5 -fold or more in rrp4-G226D cells. Statistically significant fold changes in transcript levels (Down or Up) in rrp4-G226D cells relative to RRP4 cells are color coded (1.5-2 FC [blue]; 2-4 FC [orange]; $\geq 4$ FC [purple]; $P$-value adjusted $\leq 0.05$ ). Transcripts that were subsequently validated by RT-qPCR are labeled. (C) Pie charts of the percentages of different RNA classes within the 860 Up and 802 Down transcripts in rrp4G226D cells reveal that increased transcripts are predominantly ncRNAs (CUTs; SUTs) and decreased transcripts are predominantly mRNAs. The RNA classes identified include messenger RNA (mRNA), small nuclear RNA (snRNA), small nucleolar RNA (snoRNA), transfer RNA (tRNA), cryptic unstable transcripts (CUTs; small, noncoding RNA), stable unannotated transcripts (SUTs; small, noncoding RNA), and other noncoding RNA (ncRNA; e.g., TLC1), (D) Gene ontology (GO) analysis for biological process in the Up and Down transcripts in rrp4-G226D cells reveals that ncRNA processing is significantly represented in the Up transcripts and translation is significantly represented in the Down transcripts. GO analysis was performed on coding (mRNA) and noncoding RNA (tRNAs, snoRNAs, and snRNAs) classes using the YeastEnrichr web server (Chen et al. 2013; Kuleshov et al. 2016, 2019). Gray bars represent the statistical significance of the biological process categories computed using combined score listed (log of the $P$-value from the Fisher exact test multiplied by the $z$-score of the deviation from the expected rank).

as these coding RNAs are not affected in $\operatorname{rrp} 40-$ W195R cells (Fig. 7C). The PTH4 mRNA is significantly increased in rrp40-W195R cells compared to RRP40 control, as observed in rrp4G226D cells; however, the magnitude of the change detected was quite different. With respect to the NNS components, the NRD1 steady-state level changes to a similar extent in both rrp4-G226D and rrp40-W195R cells compared to control; however, the significant increase in the NAB3 steady-state level occurs only in rrp4G226D cells (Fig. 7D).

\section{The rrp4-G226D mutant shows genetic interactions with nuclear RNA exosome cofactors}

The specificity of the RNA exosome for different RNA substrates is conferred by several interacting cofactors, which have been most extensively characterized in budding yeast (Schneider and Tollervey 2013; Zinder and Lima 2017). As depicted in Figure 8A, the exosome cofactor Rrp47 interacts with and stabilizes the exoribonuclease Rrp6, and the cofactor Mpp6 directly interacts with the nuclear RNA exosome (Schuch et al. 2014; Wasmuth et al. 2014, 2017). To determine whether the rrp4G226D variant exhibits genetic interactions with RNA exosome cofactor mutants, we deleted the nonessential, nuclear exosome cofactor genes MPP6, RRP47 and RRP6 in combination with rrp4-G226D. For comparison, we also tested whether the rrp40-W195R variant shows genetic interactions with this series of mutants by deleting each gene in combination with rrp40-W195R. We examined the growth of these double mutants relative to single mutants (rrp4-G226D and rrp40-W195R) in solid media growth assays (Fig. 8). Interestingly, the rrp4-G226D mpp64, rrp4-G226D rrp6 $\Delta$, and rrp4-G226D rrp474 double mutant cells all exhibit impaired growth compared to rrp4-G226D and single mutants at $30^{\circ} \mathrm{C}$ (Fig. 8B), indicating that deletion of MPP6, 

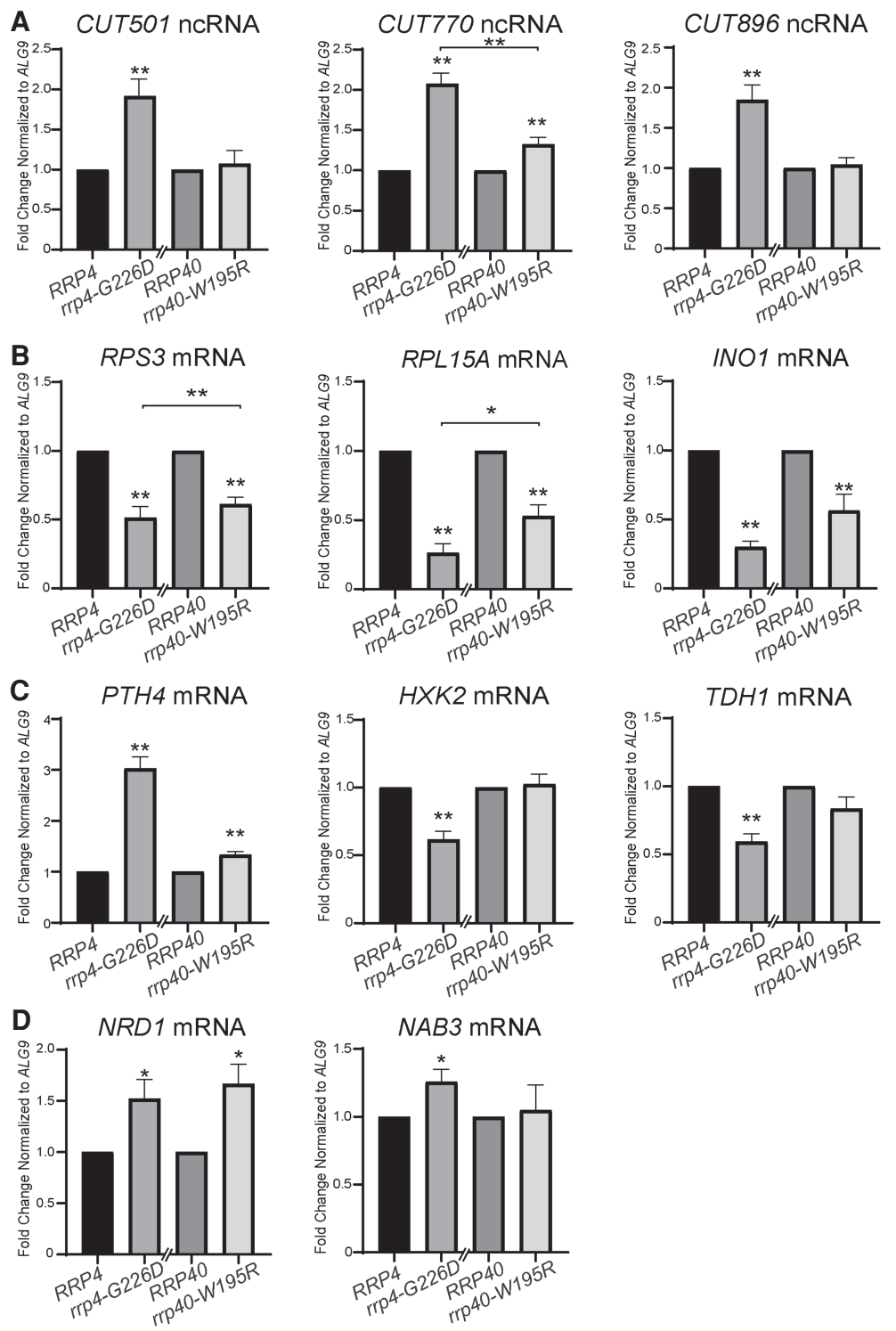

RRP47, or RRP6 exacerbates the growth defect of rrp4-G226D cells. The impaired growth of the rrp4G226D rrp6s double mutant is particularly striking. In contrast, rrp40W195R mpp6s, rrp40-W195R rrp474, and rrp40-W195R rrp6s double mutant cells do not show altered growth compared to rrp40-W195R or single mutant cells at $30^{\circ} \mathrm{C}$ (Fig. $8 \mathrm{C}$ ).

The rrp4-G226D double mutants also exhibit enhanced growth defects relative to single mutants at $37^{\circ} \mathrm{C}$. The impaired growth of the $\operatorname{rrp} 4-G 226 D$ mpp6s double mutant at $37^{\circ} \mathrm{C}$ is particularly noteworthy as loss of MPP6 does not alter cell growth at either $30^{\circ} \mathrm{C}$ or $37^{\circ} \mathrm{C}$ in single mutant cells or in double mutant rrp40-W195R cells (Fig. 8B,C). The rrp40-W195R mpp6s rrp47D and rrp40-W195R rrp6s double mutant cells exhibit impaired growth at $37^{\circ} \mathrm{C}$, though not substantially worse when compared to the impaired growth of single mutants rrp47 $\Delta$ or $\operatorname{rrp} 6 \Delta$ at $37^{\circ} \mathrm{C}$, as has been previously reported (Fig. 8B,C; Briggs et al. 1998; Mitchell et al. 2003).

\section{Rrp4 G226D has decreased association with the essential helicase Mtr4}

The nuclear exosome cofactors Mpp6 and Rrp47 and the associated exoribonuclease Rrp6 aid in recruiting the essential nuclear RNA helicase, Mtr4, to the RNA exosome (Fig. 9A; Wasmuth et al. 2017). Rrp6 and Rrp47 form a composite site that binds to the amino terminus of Mtr4, recruiting the helicase to the RNA exosome (Schuch et al. 2014). Mpp6 also tethers Mtr4 and Mtr4-containing complexes to the complex (Falk et al. 2017). Structural studies have also shown that human MTR4/MTREX and budding yeast Mtr4 directly interact with the RNA exosome by binding to a conserved region of EXOSC2/Rrp4 that also facilitates EXOSC10/Rrp6-RNA exosome interaction (Fig. 9A; Schuller et al. 2018; Weick et al. 2018). Given the negative genetic interactions observed 
A

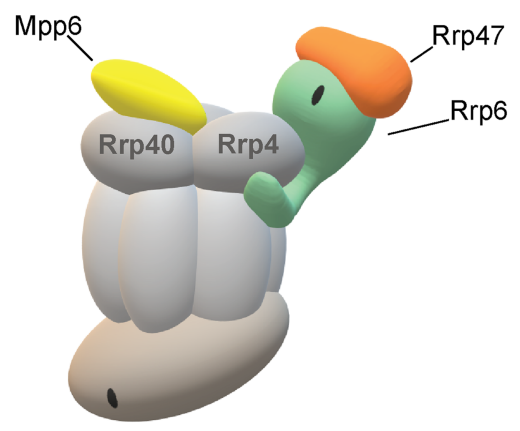

B
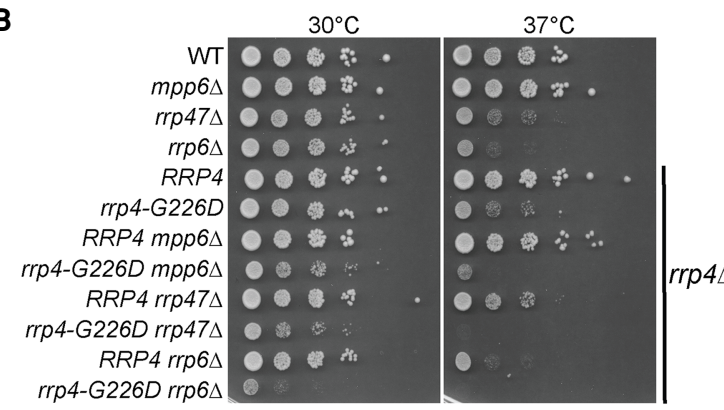

C

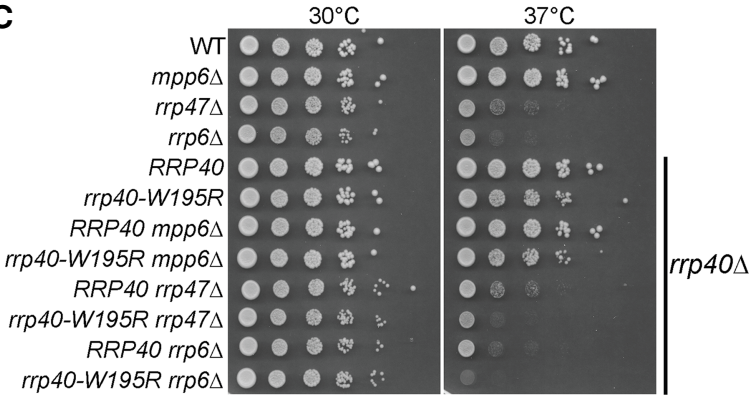

FIGURE 8. The rrp4-G226D mutant exhibits distinct negative genetic interactions with RNA exosome cofactor mutants that are not shared by the rrp40-W195R mutant. (A) Cartoon schematic of the budding yeast nuclear RNA exosome interacting with nuclear cofactors, Mpp6 and Rrp47, and the exoribonuclease Rrp6 (Schuller et al. 2018). (B) Double mutant cells containing rrp4-G226D and mpp6 $\Delta$, $\operatorname{rrp} 47 \Delta$, or rrp6 $\Delta$ show impaired growth compared to single mutants at $30^{\circ} \mathrm{C}$ and $37^{\circ} \mathrm{C}$. The double mutant cells ( $r r p 4 \Delta$ with mpp6 $\Delta$, rrp474, or rrp6 4 ) containing control RRP4 or rrp4-G226D plasmid were serially diluted, spotted onto solid media, and grown at the indicated temperatures for $3 \mathrm{~d}$. (C) Double mutant cells containing rrp40$W 195 R$ and mpp6s do not exhibit a change in growth compared to single mutants, whereas double mutant cells containing rrp40W195R and rrp47 $\Delta$ or rrp6 $\Delta$ show impaired growth compared to sin-

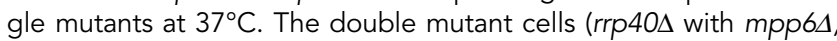
$\operatorname{rrp} 47 \Delta$, or rrp6 4 ) containing control RRP40 or $\operatorname{rrp} 40-W 195 R$ plasmid were serially diluted, spotted onto solid media, and grown at indicated temperatures for $3 \mathrm{~d}$.

between rrp4-G226D and nuclear cofactor mutants, and the binding interface between EXOSC2 and human MTR4 shown in structural studies (Weick et al. 2018), we tested whether the interaction between the RNA exosome and Mtr4 is affected in rrp4-G226D cells.

We tested for a genetic interaction between rrp4G226D and mtr4-F7A-F10A, a mutant allele of MTR4 that disrupts interactions with Rrp6/Rrp47 (Fig. 9B; Schuch et al. 2014). The rrp4-G226D mtr4-F7A-F10A double mutant cells grow similarly to $\mathrm{rrp} 4-\mathrm{G} 226 \mathrm{D}$ cells at $30^{\circ} \mathrm{C}$; however, the rrp4-G226D mtr4-F7A-F10A cells are not viable at $37^{\circ} \mathrm{C}$ (Fig. 9B). As a comparison, we performed a similar growth assay with rrp40-W195R mtr4-F7A-F10A double mutant cells and found that these cells show growth similar to $\operatorname{rrp} 40-W 195 \mathrm{R}$ cells at $30^{\circ} \mathrm{C}$ and $37^{\circ} \mathrm{C}$ (Fig. 9B). These data show that rrp4-G226D genetically interacts with a mutant allele of MTR4 that disrupts interactions with Rrp6/Rrp47.

To investigate whether the physical interaction between Mtr4 and the RNA exosome is impacted by the Rrp4 G226D variant, we performed a coimmunoprecipitation with cells that express Rrp4-Myc or Rrp4 G226D-Myc as the sole copy of Rrp4 and coexpress Mtr4-FLAG (Fig. 9C-E). The Rrp4-Myc proteins were immunoprecipitated and association with Mtr4-FLAG was assayed by immunoblotting. Mtr4-FLAG coimmunoprecipitates with Rrp4Myc but not with Rrp4 G226D-Myc (Fig. 9C). Results from three independent experiments are quantified for the amount of coisolated Mtr4-FLAG in Figure 9D. This difference in association of Mtr4-FLAG with wild-type Rrp4 versus Rrp4 G226D is not due to decreased protein levels or inefficient immunoprecipitation of Rrp4 G226D-Myc as quantitated in Figure 9E. Rather, these data demonstrate that Mtr4 association with the Rrp4 cap subunit is significantly disrupted by the Rrp4 G226D amino acid substitution (Fig. 9C,D). Combined with the genetic data (Fig. 9B), these results suggest that there is a disruption between Mtr4 and the RNA exosome complex in rrp4G226D cells, thus providing a potential molecular mechanism for the impairment in RNA exosome caused by the Rrp4 G226D amino acid substitution.

\section{DISCUSSION}

In this study, we modeled and analyzed pathogenic amino acid substitutions in the S. cerevisiae EXOSC2 ortholog, Rrp4. We generated rrp4-G58V and rrp4-G226D mutants, which correspond to the SHRF-linked mutations EXOSC2G30V and EXOSC2-G198D, respectively. Analysis of the rrp4-G58V and rrp4-G226D cells reveals that these amino acid substitutions have distinct effects on RNA exosome function. The Rrp4-G58V variant is not able to function as the sole copy of the essential Rrp4 RNA exosome cap subunit as rrp4-G58V cells are not viable. In contrast, rrp4-G226D cells show a growth defect at $37^{\circ} \mathrm{C}$. These rrp4-G226D cells show significant transcriptomic changes compared to control RRP40 cells, including increases in steady-state levels of known direct RNA exosome targets such as precursors of $5.8 \mathrm{~S}$ ribosomal RNA (rRNA), U4 small nuclear RNA (snRNA), and TLC1 telomerase RNA (Mitchell et al. 1996; Allmang et al. 1999; van Hoof et al. 2000; Davis and Ares 2006; Xu et al. 2009; Marquardt et al. 2011; 

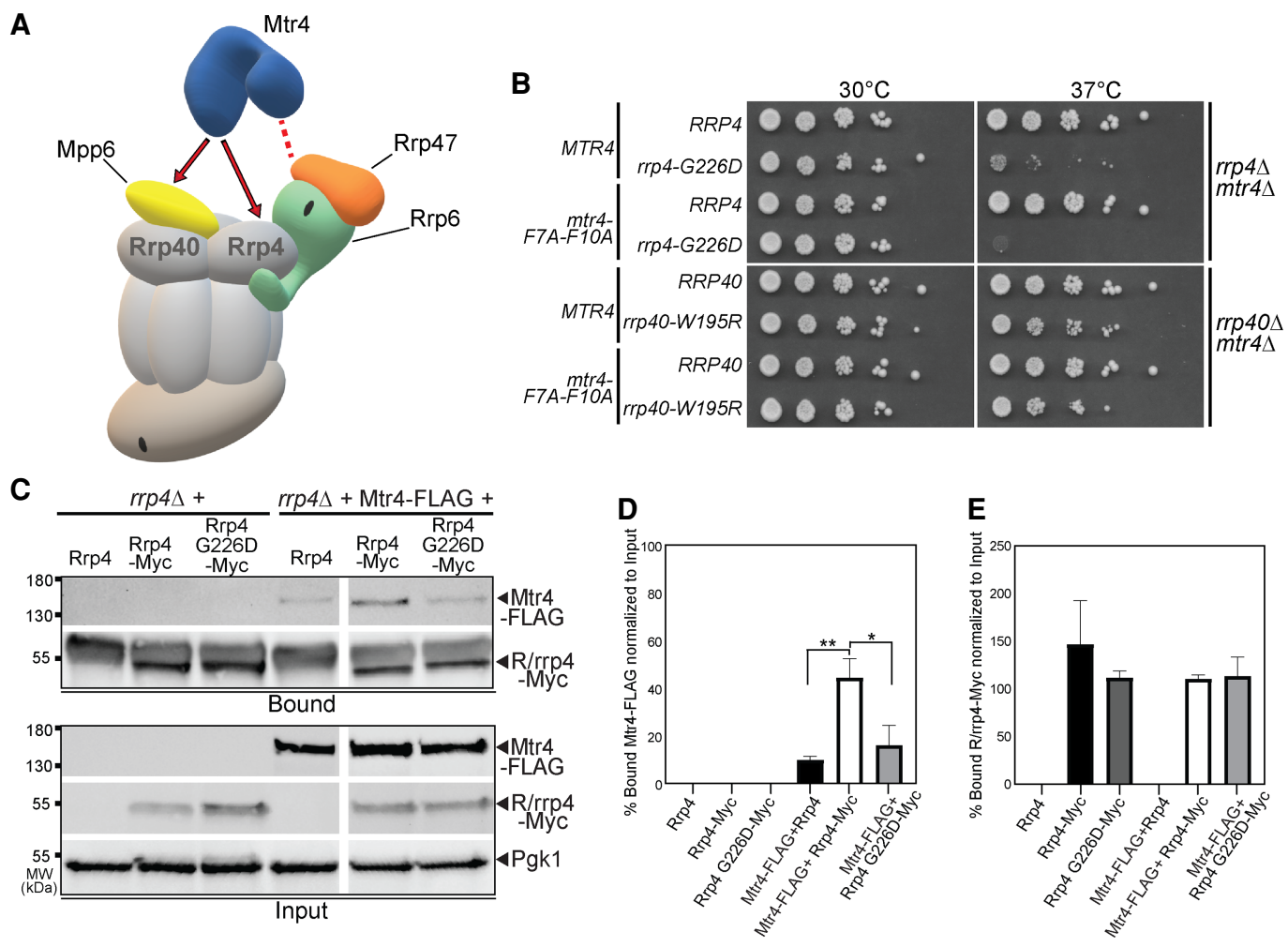

FIGURE 9. The rrp4-G226D mutant shows genetic interaction with an mtr4 mutant that is impaired for interaction with Rrp6/Rrp47 and Rrp4 G226D impairs interaction with Mtr4. (A) Cartoon of the budding yeast nuclear RNA exosome depicting the molecular interactions that the essential RNA helicase, Mtr4, makes with the RNA exosome, and exosome cofactors (Schuch et al. 2014; Falk et al. 2017; Schuller et al. 2018). The association of the amino terminus of Mtr4 with the RNA exosome is facilitated by interactions with nuclear exosome cofactors, Rrp6/Rrp47 (denoted by the dashed red line). The association of Mtr4 with the RNA exosome is also facilitated by interactions with nuclear exosome cofactor, Mpp6, which is associated with the Rrp40 exosome subunit, and the Rrp4 exosome subunit (denoted by the solid red arrows). (B) Double mutant cells containing rrp4-G226D and mtr4-F7A-F10A, an mtr4 mutant impaired for interaction with Rrp6/Rrp47, show lethality compared to the impaired growth of the single mutant rrp4-G226D at $37^{\circ} \mathrm{C}$. In contrast, double mutant cells containing rrp40-W195R and mtr4-F7A-F10A show impaired growth at $37^{\circ} \mathrm{C}$ that is similar to the single mutant rrp40-W195R, which has been described previously (Fasken et al. 2017; Gillespie et al. 2017). The rrp $4 \Delta \mathrm{mtr} 4 \Delta$ double mutant cells containing RRP4 or rrp4-G226D plasmid and rrp $40 \Delta \mathrm{mtr} 4 \Delta$ double mutant cells containing RRP40 or rrp40-W195R plasmid that also harbor MTR4 or mtr4-F7A-F10A plasmid were serially diluted, spotted onto solid media, and grown at the indicated temperatures for $3 \mathrm{~d}$. (C) The Rrp4 G226D variant shows decreased association with Mtr4. Myc-tagged Rrp4 and Rrp4 G226D protein was immunoprecipitated from rrp4 $\Delta$ cells coexpressing Rrp4-Myc and FLAG-tagged Mtr4 grown at $30^{\circ} \mathrm{C}$ using anti-Myc beads, and the amount of bound Mtr4-FLAG protein was detected by immunoblotting. The bound/input level of Mtr4-FLAG was detected with an anti-FLAG antibody, and the bound/input level of Rrp4-Myc was detected with an anti-Myc antibody. The input level of 3-phosphoglycerate kinase (Pgk1) was detected as a loading control. (D) Quantitation of the percentage of bound Mtr4-FLAG coimmunoprecipitated with Rrp4-Myc and Rrp4 G226D-Myc. Graph shows the mean percentage of bound Mtr4-FLAG from three independent experiments $(n=3)$. Error bars represent standard error of the mean. Statistical significance is denoted by asterisks ( ${ }^{*} P$-value $\leq 0.05$; ${ }^{*} P$-value $\left.\leq 0.01\right)$. (E) Quantitation of percentage of bound Rrp4-Myc and Rrp4 G226D-Myc immunoprecipitated. Error bars represent standard error of the mean. The coimmunoprecipitations were performed and quantitated as described in Materials and Methods.

Parker 2012; Schneider et al. 2012; Coy et al. 2013). RNAseq analysis of rrp4-G226D cells show broad transcriptomic changes, with predominantly increased steady-state levels of noncoding RNA CUTs and SUTs that are usually regulated by nuclear surveillance mechanisms. The Rrp4 G226D variant can assemble into the RNA exosome, but both genetic and biochemical studies suggest interactions with key RNA exosome cofactors are impaired in rrp4G226D cells. In particular, we observe decreased association of the essential helicase Mtr4 with Rrp4 G226D, suggesting a decreased interaction with this nuclear cofactor in vivo. Overall, these data suggest that the
SHRF-linked pathogenic amino acid substitutions alter the overall function of the RNA exosome in vivo, resulting in defects in nuclear surveillance that may be due to impaired interaction with Mtr4. These results provide the first in vivo model of pathogenic amino acid substitutions that occur in EXOSC2.

In assessing the molecular and functional consequences of these rrp4 variants in vivo, we first tested whether the modeled SHRF pathogenic amino acid substitutions affect protein levels and/or incorporation of the cap subunit into the RNA exosome. These data complement prior biochemical studies that used SHRF patient cells (Yang et al. 
2019). Our biochemical assays suggest that Rrp4 G58V and Rrp4 G226D are not able to incorporate into the RNA exosome complex when a wild-type copy of the cap subunit is present (Fig. 4A); however, Rrp4 G226D incorporates into the complex when no competing wild-type subunit is present (Fig. 4B). These analyses suggest that the Rrp4 variants are outcompeted by a wild-type Rrp4 for incorporation into the complex, which is similar to previous studies of rrp40-W195R cells showing that Rrp40 variants cannot incorporate into the complex in the presence of a wild-type copy of Rrp40 and are subsequently targeted by the proteasome for degradation (Fasken et al. 2017). This reported decrease in protein half-life of unincorporated subunits into the complex could explain the decrease in steady-state level of Rrp4 G58V and Rrp4 G226D in cells expressing a wild-type RRP4 copy (Fig. 3E). The lethality observed in rrp4-G58V cells when Rrp4 G58V is the sole copy of the essential cap subunit (Fig. 3A) could mean that Rrp4 G58V cannot associate with the RNA exosome, perhaps resulting in loss of functional complex in vivo. In contrast, rrp4-G226D cells are viable, but show temperature-sensitive growth.

Two of the three SHRF patients identified thus far are homozygous for the missense mutation EXOSC2-G30V ( $\mathrm{Di}$ Donato et al. 2016), suggesting that this EXOSC2 variant is able to support RNA exosome function in humans. From our structural modeling, we predict similarities between both the EXOSC2-EXOSC4 and Rrp4-Rrp41 interface (Fig. 2A,C) and a conserved stabilizing salt bridge between the two RNA exosome subunits that depends on the Gly30 residue in EXOSC2 and Gly58 residue in Rrp4 (Supplemental Fig. S2). The EXOSC2-EXOSC4 and Rrp4-Rrp41 interfaces may be differentially impacted by the valine substitution in the two eukaryotic species which could account for the differences between budding yeast rrp4-G58V cells and homozygous EXOSC2-G30V patients. Previous studies also suggest that the RNA exosome plays an important role in tissue development and human embryonic stem cell differentiation (Belair et al. 2019; Yatsuka et al. 2020). The diverse clinical presentation in patients with SHRF could reflect these key developmental roles and/or different requirements in different cell types. Thus, the differential effects observed between budding yeast rrp4-G58V cells and EXOSC2-G30V patients could be indicative of differences in developmental time points or requirements between the two eukaryotes. Integrating additional disease models across other systems will be required to define how pathogenic missense mutations differentially impact RNA exosome function in a tissuespecific manner, leading to diverse disease pathologies.

RNA-seq analysis of the rrp4-G226D mutant cells revealed a broad spectrum of RNA classes that are altered in these mutant cells. The majority of the significantly increased transcripts in rrp4-G226D cells are comprised of the noncoding RNAs, CUTs, and SUTs (65\% of all up tran- scripts with $\mathrm{FC} \geq+1.5)$. As CUTs and SUTs are stabilized in RNA exosome mutants and cross-link to the RNA exosome (Wyers et al. 2005; Davis and Ares 2006; Gudipati et al. 2012; Schneider et al. 2012), we suggest that the elevated CUTs and SUTs observed in rrp4-G226D cells are due to impaired nuclear exosome function due to the Rrp4 G226D substitution. In contrast, the overwhelming majority of the significantly decreased transcripts are mRNAs (90\% of all down transcripts with $\mathrm{FC} \geq-1.5$ ), with the most significantly decreased transcript being INO1 mRNA. Previous studies have shown that INO1 mRNA associates with the catalytic Dis3/Rrp44 subunit of the RNA exosome in budding yeast as determined by UV cross-linking and analysis of cDNA (CRAC) (Delan-Forino et al. 2017). This published data set also reports physical interaction of Dis3/Rrp44 with HXK2, TDH1, RPL15A, and RPS3 mRNAs (Delan-Forino et al. 2017); other RNAs decreased in our RNA-seq analysis. The authors of this study suggested that these mRNA targets could be rapidly turned over in the cytoplasm by the RNA exosome, but our results show a decrease in these mRNAs, rather than the increase predicted if these transcripts were rapidly degraded by the RNA exosome. Notably, many ribosomal protein gene (RPG) mRNAs are decreased in rrp4-G226D cells and GO analysis of the decreased transcripts revealed cytoplasmic translation to be the most significantly affected biological process (Fig. 5D). Consistent with these data, decreases in RPG mRNAs have also been observed in rrp6 4 mutant cells, (Fox et al. 2015). Decreases of these specific mRNAs in rrp4-G226D cells could reflect dysregulation of the cytoplasmic RNA exosome in rrp4-G226D cells; however, it is not clear how these mRNA targets that physically associate with the catalytic subunit Rrp44 are decreased in rrp4-G226D cells. Overall, these data demonstrate that the Rrp4 G226D variant could alter both nuclear and cytoplasmic roles of the RNA exosome.

Many of the decreased mRNA transcripts observed in rrp4-G226D cells could result from indirect effects, reflecting cellular changes that occur when the function of the RNA exosome is compromised, leading to numerous downstream changes. Previous work in Drosophila melanogaster that used RNAi to deplete Rrp4 identified decreased levels of several transcripts encoding autophagy proteins (Yang et al. 2019). The authors postulated that defective autophagy could contribute to SHRF pathology (Yang et al. 2019). In our RNA-seq analysis of rrp4G226D cells, we identified 16 autophagy transcripts that were decreased -1.5 -fold $(P<0.05)$ (Supplemental Fig. $S 4)$, which is consistent with observations in this previous study. Further studies will be required to assess whether rrp4-G226D cells have impaired autophagy as well as to determine whether these mRNA transcripts are direct targets of regulation by the RNA exosome.

Both genetic and biochemical assays suggest that defects in the RNA exosome function in rrp4-G226D cells 
could be due to disrupted cofactor interactions, particularly decreased association with the essential RNA helicase Mtr4. Human structural studies have shown that MTR4/ MTREX binds directly not only to MPH6/MPP6 but also to a conserved region of EXOSC2 (Weick et al. 2018). Perturbance of the MTR4/MTREX/Mtr4-RNA exosome interaction in humans and budding yeast could also impact association of the NEXT complex or TRAMP complex with the RNA exosome, thus affecting nuclear RNA quality control of several RNA classes, including processing of telomerase RNA, and degradation of cryptic ncRNAs (Houseley and Tollervey 2009). The increased levels of CUTs, SUTs, and precursors of U4 snRNA and TLC1 observed in rrp4-G226D cells further lends support to suggest that the Mtr4-RNA exosome interaction is impaired by Rrp4 G226D. This finding is consistent with a previous study that used a structural and biochemical approach to study the budding yeast nuclear RNA exosome and the consequences of the pathogenic EXOSC3 W238R variant linked to PCH1b in the human RNA exosome (Falk et al. 2017). This study showed that substitution of Arg for Trp at position 238 (W238R) in EXOSC3, corresponding to Rrp40 W195R modeled in yeast, impaired the interaction with MPH6/MPP6. Taken together, our analysis of Rrp4 variants and the previous study of Rrp40 variants suggest that pathogenic amino acid substitutions in cap subunits could impair interactions with RNA exosome cofactors, suggesting a molecular mechanism that could underlie impaired RNA exosome function in vivo.

This study identified several in vivo consequences resulting from the Rrp4 G226D amino acid substitution. Biochemical, genetic, and RNA analyses show that rrp4G226D cells have impaired RNA exosome function, broad transcriptomic changes and defects in RNA exosome cofactor interactions. When we compare these functional and molecular consequences in rrp4-G226D cells to rrp40-W195R cells we see some similarities and some differences. Both exosomopathy mutant models show growth defects, though rrp4-G226D cells show a more severe growth phenotype (Fig. 3B). In addition, both exosomopathy mutant models share changes in steady-state levels of some transcripts (Fig. 7B), but some transcripts show statistically significant changes only in rrp4-G226D cells. Genetic analyses also suggest that rrp4-G226D and rrp40-W195R mutants have similar negative genetic interactions with key nuclear exosome cofactor mutants (Fig. 8). However, we also detect a negative genetic interaction between rrp4-G226D and an mtr4 mutant that is not observed with rrp40-W195R. Based on these observations, two possibilities present themselves. One possibility is that missense mutations in RRP4 and RRP40 have distinct functional consequences for RNA exosome activity, which would be consistent with the distinct clinical presentations in patients with these pathogenic variants. Alternatively, the rrp4-G226D allele may simply be a stronger allele than rrp40-W195R. A more extensive, comparison of additional exosomopathy mutant alleles will be required to distinguish between these two possibilities.

Utilizing the yeast genetic model system, we have begun to elucidate the functional consequences that result from the pathogenic amino acid substitutions in EXOSC2 in SHRF patients. By modeling these mutations in the corresponding RRP4 gene, we have generated a system that can be used to understand how pathogenic amino acid substitutions impact the function of the RNA exosome. This study also adds to the growing collection of in vivo RNA exosomopathy mutant models that can be compared to one another to define the in vivo consequences resulting from each mutation. For several RNA exosomopathies, including SHRF syndrome, the patient population is quite small, making analysis of patient tissue samples challenging if not impossible. Our findings presented here can be integrated into the body of work describing the SHRF EXOSC2 mutations, further expanding our understanding of the unique disease pathology. Future comparative in vivo analysis of exosomopathy models will allow for deeper understanding of how diverse clinical symptoms are linked to changes in a single molecular machine. Furthermore, this type of in vivo comparison may shed light on the basic biology of the RNA exosome, as many questions still exist regarding RNA substrate targeting and regulation of this essential molecular machine. This study not only provides the first in vivo study that models EXOSC2 mutations identified in SHRF patients, but also provides a platform for the first direct comparison of the consequences of pathogenic missense mutations in genes encoding cap subunits of the RNA exosome.

\section{MATERIALS AND METHODS}

\section{Chemicals and media}

All chemicals were obtained from Sigma-Aldrich, United States Biological, or Fisher Scientific unless otherwise noted. All media were prepared by standard procedures (Adams et al. 1997).

\section{Protein structure analysis}

We used the cryo-EM structure (PDB 6D6R) of the human nuclear RNA exosome at $3.45 \AA$ resolution (Weick et al. 2018) and the cryo-EM structure (PDB 6FSZ) of the budding yeast nuclear RNA exosome at $4.6 \AA$ (Schuller et al. 2018). Structural modeling was performed using the PyMOL viewer (The PyMOL Molecular Graphics System, Version 2.0 Schrödinger, LLC). The mCSMPP12 (Rodrigues et al. 2019), Polymorphism Phenotyping v2 (PolyPhen-2) (Adzhubei et al. 2010), Protein Variation Effect Analyzer (PROVEAN) (Choi 2012; Choi et al. 2012), and SNAP-2 (Hecht et al. 2015) webservers were used for predicting the effects of the EXOSC2 mutations on protein stability and function. 


\section{Saccharomyces cerevisiae strains and plasmids}

All DNA manipulations were performed according to standard procedures (Sambrook et al. 1989). S. cerevisiae strains and plasmids used in this study are listed in Supplemental Table S1. The rrp4 $\Delta$ (yAV1103) and rrp40 $\Delta$ (yAV1107) strains were previously described (Schaeffer et al. 2009; Losh 2018). The RRP43-TAP (ACY2788) strain was obtained from Horizon Discovery Biosciences Limited and was previously described (Ghaemmaghami et al. 2003). The RRP43-TAP rrp4A (ACY2803) strain was constructed by deletion of the genomic RRP4 ORF in the RRP43-TAP (ACY2788) strain containing a RRP4 URA3 maintenance plasmid by homologous recombination using a RRP4-UTR neoMX4 PCR product. The $\operatorname{rrp} 4 \Delta$ mpp6 $\Delta$ (ACY2471), rrp4 $\Delta$ rrp47 $\Delta$ (ACY2474), rrp4 4 rrp6s (ACY2478) strains and the rrp404 mpp6s

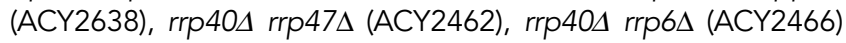
strains were constructed by deletion of the MPP6, RRP47, and

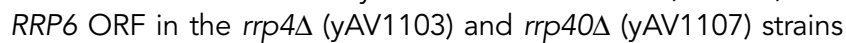
by homologous recombination using MPP6-, RRP47-, or RRP6UTR natMX4 PCR products. The rrp4 $m$ tr4 $\Delta$ (ACY2536) and

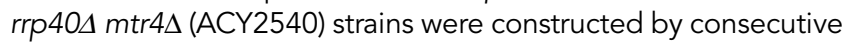
deletion of the genomic MTR4 ORF and RRP4 ORF or RRP40 ORF in a wild-type (W303) strain harboring a [-MTR4; RRP4; URA3] (pAC3714) or [MTR4; RRP40; URA3] (pAC3713) maintenance plasmid by homologous recombination using MTR4-UTR natMX4 and RRP4-UTR or RRP40-UTRneoMX4 PCR products. Construc-

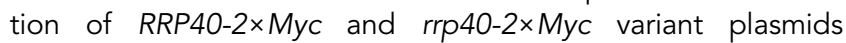
(pAC3161, pAC3162, and pAC3259) was reported previously (Fasken et al. 2017). The RRP4-2×Myc LEU2 CEN6 (pAC3474) plasmid was constructed by PCR amplification of the endogenous promoter, 5'-UTR and ORF of the RRP4 gene from S. cerevisiae genomic DNA and cloning into pRS315 plasmid containing a carboxy-terminal 2xMyc tag and the ADH1 3'-UTR (Sikorski and Hieter 1989). The rrp4-G58V-2×Myc (pAC3476) and rrp4G226D-2×Myc (pAC3477) plasmids were generated by site-directed mutagenesis of the RRP4-2×Myc (pAC3474) plasmid using oligonucleotides containing the SHRF syndrome-linked G58V and G226D missense mutations and the QuikChange II Site-Directed Mutagenesis Kit (Agilent). The untagged RRP4/ rrp4-G226D (pAC3656, pAC3659) and RRP40/rrp40-W195R (pAC3652, pAC3655) plasmids and Myc-tagged RRP4/rrp4G226D (pAC3669, pAC3672) plasmid containing native 3'-UTRs were generated by excision of the $2 \times M y c-A D H 1 \quad 3^{\prime}-U T R$ from each RRP4/40-Myc LEU2 CEN6 plasmid by restriction digestion and cloning of the native RRP4 $3^{\prime}$-UTR, RRP4O $3^{\prime}$-UTR, or $2 \times$ Myc-RRP4 3'-UTR PCR product into the relevant plasmid using NEBuilder HiFi Assembly (New England BioLabs). The -MTR4 HIS3 CEN6 (pAC4096) plasmid was constructed by PCR amplification of the endogenous promoter, 5'-UTR, ORF, and 3'UTR of the MTR4 gene from $S$. cerevisiae genomic DNA and cloning into pRS313 (Sikorski and Hieter 1989). The mtr4-F7A-F10A (pAC4099) plasmid was generated by site-directed mutagenesis of the MTR4 HIS CEN6 plasmid (pAC4096) using oligonucleotides containing the F7A and F10A missense mutations and the QuikChange II Site-Directed Mutagenesis Kit (Agilent). The MTR4-2×FLAG (pAC3719) plasmid was constructed by PCR amplification of the MTR4 promoter-5'-UTR-ORF (No Stop) and 2×FLAG-native MTR4 3'-UTR using MTR4 template plasmid (pAC2897 [Fasken et al. 2011]) and oligonucleotides encoding $2 \times F L A G$ epitopes and cloning into the pRS313 plasmid (Sikorski and Hieter 1989).

\section{Saccharomyces cerevisiae transformations and growth assays}

All yeast transformations were performed according to the standard Lithium Acetate (LiOAc) protocol (Burke et al. 2000). Cells were grown overnight to saturation in a $30^{\circ} \mathrm{C}$ incubator in liquid YEPD (1\% yeast extract, $2 \%$ peptone, $2 \%$ dextrose, in distilled water). Cell concentrations were normalized to an $\mathrm{OD}_{600}=0.4$ in $10 \mathrm{~mL} \mathrm{YEPD} \mathrm{and} \mathrm{incubated} \mathrm{at} 30^{\circ} \mathrm{C}$ for $5 \mathrm{~h}$. The cells were washed with TE/LiOAC and resuspended in TE/LiOAc to a concentration of $2 \times 10^{9}$ cells $/ \mathrm{mL}$. To the cells $(50 \mu \mathrm{L})$, plasmid DNA, singlestranded carrier DNA $(5 \mu \mathrm{L})$, and PEG/TE/LiOAc $(300 \mu \mathrm{L})$ were added and cells were agitated for 30 mins at $30^{\circ} \mathrm{C}$. DMSO $(35 \mu \mathrm{L})$ was added and the cells were heat shocked at $42^{\circ} \mathrm{C}$ for $15 \mathrm{~min}$. The cells were washed and plated onto selective media.

To test the in vivo function of the rrp4 variants that model the EXOSC2 variants in SHRF syndrome, a standard plasmid shuffle assay was used. The $\operatorname{rrp} 4 \Delta$ (yAV1103) cells containing a RRP4 URA3 maintenance plasmid and transformed with vector (pRS315), RRP4-2×Myc (pAC3474), rrp4-G8A$2 \times$ Myc (pAC3476), or rrp4-G226D-2×Myc (pAC3477) plasmid were grown overnight and serially diluted and spotted onto Ura- Leu $^{-}$minimal media plates, which select for cells that contain both the RRP4 URA3 maintenance plasmid and the RRP4/rrp4 LEU2 plasmid, and 5-FOA Leu ${ }^{-}$minimal media plates, which select for cells that lack the RRP4 URA3 maintenance plasmid and contain only the RRP4/rrp4 LEU2 plasmid. The plates were incubated at $30^{\circ} \mathrm{C}$ and $37^{\circ} \mathrm{C}$ for $2 \mathrm{~d}$.

The in vivo function of the rrp4-G226D variant and the rrp40W195R variant was assessed in growth assays on solid media and in liquid culture. For growth on solid media, rrp4 $\Delta$ (yAV1103) cells containing only RRP4 (pAC3656) or rrp4-G226D (pAC3659) and rrp40S (yAV1107) cells containing only RRP40 (pAC3652) or rrp40-W195R (pAC3655) were grown in $2 \mathrm{~mL}$ $\mathrm{Leu}^{-}$minimal media overnight at $30^{\circ} \mathrm{C}$ to saturation. Cell concentrations were normalized to an $\mathrm{OD}_{600}=0.5$, serially diluted in 10 fold dilutions, spotted onto $\mathrm{Leu}^{-}$minimal media plates, and grown at $25^{\circ} \mathrm{C}, 30^{\circ} \mathrm{C}$, and $37^{\circ} \mathrm{C}$ for $2-3 \mathrm{~d}$. For growth in liquid culture, cells were grown in $2 \mathrm{~mL} \mathrm{Leu}^{-}$minimal media overnight at $30^{\circ} \mathrm{C}$ to saturation, diluted to an $\mathrm{OD}_{600}=0.01$ in $\mathrm{Leu}^{-}$minimal media in a 24-well plate, and growth at $37^{\circ} \mathrm{C}$ was monitored and recorded at $\mathrm{OD}_{600}$ in a BioTek Synergy $\mathrm{Mx}$ microplate reader with Gen5 v2.04 software over $24 \mathrm{~h}$. Technical triplicates of each strain were measured, and the average of these triplicates was calculated and graphed.

\section{Immunoblotting}

For analysis of carboxy-terminally Myc-tagged Rrp4 protein expression levels, rrp4s (yAV1103) cells expressing only Rrp4$2 \times$ Myc (pAC3669) or rrp4-G226D-2×Myc (pAC3672) were grown in $2 \mathrm{~mL} \mathrm{Leu}^{-}$minimal media overnight at $30^{\circ} \mathrm{C}$ to saturation, and $10 \mathrm{~mL}$ cultures with an $\mathrm{OD}_{600}=0.2$ were prepared and grown at $30^{\circ} \mathrm{C}$ and $37^{\circ} \mathrm{C}$ for $5 \mathrm{~h}$. Additionally, rrp4 4 (yAV1103) cells containing RRP4 URA3 maintenance plasmid and expressing Rrp4$2 \times$ Myc (pAC3474), rrp4-G58V (pAC3476), or rrp4-G226D$2 \times$ Myc (pAC3477) were grown in $2 \mathrm{~mL} \mathrm{Ura}^{-} \mathrm{Leu}^{-}$minimal media overnight at $30^{\circ} \mathrm{C}$, and $10 \mathrm{~mL}$ cultures with an $\mathrm{OD}_{600}=0.2$ were prepared and grown at $30^{\circ} \mathrm{C}$ for $5 \mathrm{~h}$. Cell pellets were collected 
by centrifugation, transferred to $2 \mathrm{~mL}$ screw-cap tubes and stored at $-80^{\circ} \mathrm{C}$. Yeast cell lysates were prepared by resuspending cell pellets in $0.3-0.5 \mathrm{~mL}$ of RIPA-2 Buffer $(50 \mathrm{mM}$ Tris- $\mathrm{HCl}$, $\mathrm{pH} 8$; $150 \mathrm{mM} \mathrm{NaCl} ; 0.5 \%$ sodium deoxycholate; $1 \%$ NP40; $0.1 \%$ SDS) supplemented with protease inhibitors (1 mM PMSF; Pierce Protease Inhibitors [Thermo Fisher Scientific]), and 300 $\mu \mathrm{L}$ of glass beads. Cells were disrupted in a Mini-Beadbeater 16 Cell Disrupter (BioSpec) for $4 \times 1 \mathrm{~min}$ at $25^{\circ} \mathrm{C}$ with $1 \mathrm{~min}$ on ice between repetitions, and then centrifuged at $16,000 \mathrm{~g}$ for $15 \mathrm{~min}$ at $4{ }^{\circ} \mathrm{C}$. Protein lysate concentration was determined by Pierce BCA Protein Assay Kit (Life Technologies). Whole cell lysate protein samples $(40 \mu \mathrm{g})$ in reducing sample buffer $(50 \mathrm{mM}$ Tris $\mathrm{HCl}, \mathrm{pH}$ 6.8; $100 \mathrm{mM}$ DTT; $2 \%$ SDS; $0.1 \%$ Bromophenol Blue; $10 \%$ Glycerol) were resolved on 4\%-20\% Criterion TGX Stain-Free Precast Polyacrylamide Gels (Bio-Rad), transferred to nitrocellulose membranes (Bio-Rad), and Myc-tagged Rrp4 proteins were detected with anti-Myc monoclonal antibody 9B11 (1:2000; Cell Signaling). The 3-phosphoglycerate kinase (Pgk1) protein was detected using anti-Pgk1 monoclonal antibody (1:30,000; Invitrogen) as a loading control.

\section{Quantitation of immunoblotting}

The protein band intensities/areas from immunoblots were quantitated using ImageJ v1.4 software (National Institutes of Health, MD; http://rsb.info.nih.gov/ij/) and mean fold changes in protein levels were calculated in Microsoft Excel (Microsoft Corporation). To quantitate the mean fold change in Rrp4 G226D-Myc variant level relative to wild-type Rrp4-Myc level in rrp4 $\Delta$ cells grown at $30^{\circ} \mathrm{C}$ and $37^{\circ} \mathrm{C}$ from three immunoblots (Fig. 3D) or the fold change in rrp4-G58V-Myc and rrp4-G226D-Myc level in rrp4 $\Delta$ cells containing untagged RRP4 from three immunoblots (Fig. 3E), R/rrp4-Myc intensity was first normalized to loading control Pgk1 intensity and then normalized to wild-type Rrp4-Myc intensity at $30^{\circ} \mathrm{C}$ or $37^{\circ} \mathrm{C}$ for each immunoblot. The mean fold change in R/rrp4-Myc level relative to Rrp4-Myc and standard error of the mean are graphed in Figure $3 \mathrm{~F}$ and $\mathrm{G}$. To quantitate the mean percent bound of Mtr4-FLAG coimmunoprecipitated with Rrp4Myc or Rrp4 G226D-Myc (Fig. 9C), background intensity of Mtr4-FLAG was subtracted from the bound Mtr4-FLAG intensity. Then bound and input Mtr4-FLAG intensity was normalized to input Pgk1 intensity. Percent bound was calculated by dividing normalized bound Mtr4-FLAG by normalized input Mtr4-FLAG. To quantitate the mean percent bound Rrp4-Myc or Rrp4 G226DMyc that immunoprecipitates (Fig. 9C), bound and input R/rrp4Myc was normalized to input Pgk1 intensity. Percent bound was calculated by dividing normalized bound R/rrp4-Myc intensity by normalized input R/rrp4-Myc intensity. Mean percent bound Mtr4-FLAG and R/rrp4-Myc and standard error of the mean are graphed in Figure 9D and E.

\section{Northern blotting}

For analysis of $5.8 \mathrm{~S}$ pre-rRNA processing-detection of $7 \mathrm{~S}$ prerRNA and processing intermediates-in rrp4 and rrp40 mutant

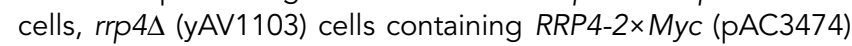
or rrp4-G226D-2×Myc (pAC3477) and rrp404 (yAV1107) cells containing RRP40-2×Myc (pAC3161), rrp40-G8A-2×Myc (pAC3162), or rrp40-W195R-2×Myc (pAC3259) were grown in 2
$\mathrm{mL} \mathrm{Leu}^{-}$minimal media overnight at $30^{\circ} \mathrm{C}, 10 \mathrm{~mL}$ cultures with an $\mathrm{OD}_{600}=0.4$ were prepared and grown at $37^{\circ} \mathrm{C}$ for $5 \mathrm{~h}$. Cells were collected by centrifugation $(2163 \mathrm{~g})$, transferred to $2 \mathrm{~mL}$ screw cap tubes and stored at $-80^{\circ} \mathrm{C}$. Total RNA from cells was resolved on a Criterion TBE-Urea Polyacrylamide Gel (Bio-Rad), blotted to a nylon membrane and membrane was probed with radiolabeled 5.8S-ITS2 rRNA (boundary) oligonucleotide (AC4211/ Probe 020-5'-TGAGAAGGAAATGACGCT) to detect 7S prerRNA and intermediates and stained with methylene blue stain to visualize $5.8 \mathrm{~S}$ rRNA as a loading control. Total RNA $(5 \mu \mathrm{g})$ was mixed with equal volume of RNA loading dye $(1 \times \mathrm{TBE} ; 12 \%$ Ficoll; 7M Urea; 0.01 bromophenol blue; $0.02 \%$ xylene cyanol) and resolved on 10\% TBE-Urea Polyacrylamide Gel in $1 \times$ TBE at $200 \mathrm{~V}$ for $1.5 \mathrm{~h}$. RNA was transferred to Hybond-N+ nylon membrane (Amersham, GE Healthcare) at $15 \mathrm{~V}$ for $100 \mathrm{~min}$ in $1 \times \mathrm{TBE}$ and cross-linked to membrane with UV light (120,000 $\mu$ Joules) using UV Stratalinker 2400 (Stratagene). The membrane was incubated in Rapid-hyb hybridization buffer (Amersham, GE healthcare) at $37^{\circ} \mathrm{C}$ for $1 \mathrm{~h}$. DNA oligonucleotide (100 ng) was $5^{\prime}$-end labeled with [ $\gamma$-P32]-ATP (PerkinElmer) using polynucleotide kinase (New England Biolabs) at $37^{\circ} \mathrm{C}$ for $30 \mathrm{~min}$. [P32]Labeled oligonucleotide probe was purified through a G25 microspin column (GE Healthcare), heated at $100^{\circ} \mathrm{C}$ for $5 \mathrm{~min}$, and added to hybridization buffer. The oligonucleotide probe was hybridized to membrane in hybridization buffer at $37^{\circ} \mathrm{C}$ overnight. Following removal of hybridization buffer, membrane was rinsed twice in $5 \times \mathrm{SSPE} ; 0.1 \% \mathrm{SDS}$ at $25^{\circ} \mathrm{C}$ and washed twice in $0.5 \times$ SSPE; $0.1 \%$ SDS at $37^{\circ} \mathrm{C}$ for 20 min each. The membrane was exposed to phosphoscreen overnight and imaged using Typhoon FLA 7000 phosphorimager (GE Healthcare).

\section{Total RNA isolation}

Total RNA from S. cerevisiae rrp4 and rrp40 mutant cells was isolated using TRIzol (Invitrogen) for qRT-PCR and northern blotting and MasterPure Yeast RNA Purification Kit (Epicentre, Lucigen) for RNA-seq. S. cerevisiae cells were grown in $2 \mathrm{~mL} \mathrm{Leu}{ }^{-}$minimal media overnight at $30^{\circ} \mathrm{C}$ to saturation. Cultures were diluted in $10 \mathrm{~mL} \mathrm{Leu}{ }^{-}$minimal media to an $\mathrm{OD}_{600}=0.2$ and grown for $5 \mathrm{~h}$ at $37^{\circ} \mathrm{C}$. Cells were pelleted by centrifugation, transferred into $2 \mathrm{~mL}$ screw cap tubes, and stored at $-80^{\circ} \mathrm{C}$. To prepare total RNA using TRIzol, cells were resuspended in $1 \mathrm{~mL}$ TRIzol (Invitrogen) with $300 \mu \mathrm{L}$ of glass beads. Cell samples were disrupted in a BioSpec Mini-Beadbeater 16 Cell Disrupter for $2 \mathrm{~min}$ at $25^{\circ} \mathrm{C}$. For each sample, $100 \mu \mathrm{L}$ of 1 -bromo-3-chloropropane $(B C P)$ was added, sample was vortexed for $15 \mathrm{sec}$, and incubated at $25^{\circ} \mathrm{C}$ for $2 \mathrm{~min}$. The sample was centrifuged at $16,300 \mathrm{~g}$ for $8 \mathrm{~min}$ at $4^{\circ} \mathrm{C}$, and the upper layer was transferred to a fresh microfuge tube. RNA was precipitated with $500 \mu \mathrm{L}$ isopropanol and sample was vortexed for $10 \mathrm{sec}$ to mix. Total RNA was pelleted by centrifugation at $16,300 \mathrm{~g}$ for $8 \mathrm{~min}$ at $4^{\circ} \mathrm{C}$. The RNA pellet was washed with $1 \mathrm{~mL} 75 \%$ ethanol, centrifuged at $16,300 \mathrm{~g}$ for $5 \mathrm{~min}$ at $4^{\circ} \mathrm{C}$, and air-dried for $15 \mathrm{~min}$. Total RNA was resuspended in $50 \mu \mathrm{L}$ diethylpyrocarbonate (DEPC, Sigma)-treated water and stored at $-80^{\circ} \mathrm{C}$. Total RNA was prepared using MasterPure Yeast RNA Purification Kit (Epicentre, Lucigen) according to manufacturer's protocol. Total RNA was resuspended in $50 \mu \mathrm{L}$ DEPC-treated water and stored at $-80^{\circ} \mathrm{C}$. 


\section{qRT-PCR}

All oligonucleotides used in this study are summarized in Supplemental Table S2. For analysis of steady-state RNA levels using quantitative PCR, three independent biological replicates of rrp4 4 (yAV1103) cells containing only RRP4 (pAC3656) or rrp4G226D (pAC3659) and rrp404 (yAV1107) cells containing only RRP40 (pAC3652) or rrp40-W195R (pAC3655) were grown in 2 $\mathrm{mL}$ Leu $^{-}$minimal media overnight at $30^{\circ} \mathrm{C}, 10 \mathrm{~mL}$ cultures with an $\mathrm{OD}_{600}=0.2$ were prepared and cells were grown at $37^{\circ} \mathrm{C}$ for $5 \mathrm{~h}$. Total RNA was isolated from cell pellets and $1 \mu \mathrm{g}$ of total RNA was reverse transcribed to first strand cDNA using the M-MLV Reverse Transcriptase (Invitrogen) according to manufacturer's protocol. Quantitative PCR was performed on technical triplicates of cDNA (10 ng) from three independent biological replicates using gene specific primers $(0.5 \mathrm{mM}$; Supplemental Table S2), QuantiTect SYBR Green PCR master mix (Qiagen) on a StepOnePlus Real-Time PCR machine (Applied Biosystems; Tanneal $=55^{\circ} \mathrm{C}, 44$ cycles). ALG9 was used as an internal control. The mean RNA levels were calculated by the $\Delta \Delta \mathrm{Ct}$ method (Livak and Schmittgen 2001). Mean levels of RNA calculated in mutant cells are normalized to mean levels in wild-type cells and converted and graphed as an RNA fold change relative to wild-type. All primers used are summarized in Supplemental Table S2.

\section{RNA-seq analysis}

RNA-seq was performed on three independent biological repli-

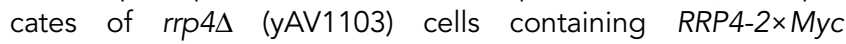
(pAC3474) or rrp4-G226D-2×Myc (pAC3477) as the sole copy of RRP4 grown at $37^{\circ} \mathrm{C}$. Cells were grown in $2 \mathrm{~mL} \mathrm{Leu}^{-}$minimal media overnight at $30^{\circ} \mathrm{C}$, diluted to an $\mathrm{OD}_{600}=0.4$ in $10 \mathrm{~mL}$ $\mathrm{Leu}^{-}$minimal media, grown at $37^{\circ} \mathrm{C}$ for $5 \mathrm{~h}$, and collected and stored at $-80^{\circ} \mathrm{C}$. Total RNA was isolated, rRNA was depleted, and stranded cDNA libraries were prepared using TruSeq Total RNA Stranded Library Prep kit (Illumina). Paired-end sequencing of the cDNA libraries was performed on a HiSeq4000 instrument $(2 \times 150$ cycles) at Frederick National Laboratory for Cancer Research (FNLCR) at the CCR Sequencing Facility, $\mathrm{NCl}, \mathrm{NIH}$. The RRP4 samples yielded an average of $28,890,739$ pass filter reads and the rrp4-G226D samples yielded an average of $34,644,683$ pass filter reads, with a base call quality of $94 \%$ of bases with Q30 and above. The reads were mapped to the S. cerevisiae S288C genome assembly R64-1-1, annotated with CUTs and SUTs (Xu et al. 2009), using the STAR RNA-seq aligner (v2.7.5b [Dobin et al. 2012]). The reads per gene feature were counted using featureCounts (v1.6.4+galaxy2 [Liao et al. 2014]). Differential gene expression analysis on raw read counts was performed using DESeq2 (v2.11.40.6+galaxy1 [Love et al. 2014]) to identify genes significantly changed ( $P$-value $<0.05, \geq 1.5$ fold change) in rrp4G226D samples relative to RRP4 samples. Principal component analysis (PCA) on raw read counts was also performed using DESeq2. A volcano plot of differential gene expression data was produced in Prism 8 (Graphpad Software). Piecharts of RNA class percentages in significantly altered genes were generated in Microsoft Excel for Mac (Microsoft Corp.). Gene Ontology (GO) analysis on significantly altered genes for Biological Process category was performed using the YeastEnrichr webserver (http://amp.pharm.mssm.edu/YeastEnrichr/ [Kuleshov et al. 2019]).

\section{Genetic interaction analysis}

To test genetic interactions between rrp4-G226D or rrp40W195R and RNA exosome cofactor/subunit deletion mutants,

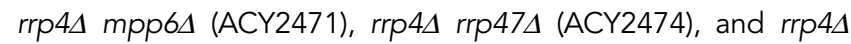
rrp64 (ACY2478) cells containing only RRP4 (pAC3656) or rrp4G226D (pAC3659) and rrp404 mpp64 (ACY2638), rrp404

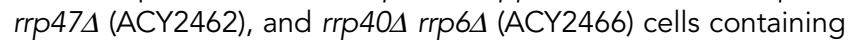
only RRP40 (ACY3652) or rrp40-W195R (ACY3655) were grown in $2 \mathrm{~mL} \mathrm{Leu}^{-}$minimal media overnight at $30^{\circ} \mathrm{C}$ to saturation, serially diluted, and spotted on $\mathrm{Leu}^{-}$minimal media plates. The plates were incubated at $30^{\circ} \mathrm{C}$ and $37^{\circ} \mathrm{C}$ for $3 \mathrm{~d}$.

To test genetic interactions between rrp4-G226D or rrp40-

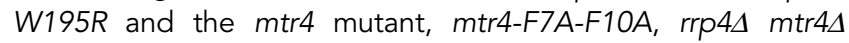
(ACY2536) cells containing the [MTR4; RRP4; URA3] (pAC3714) maintenance plasmid were transformed with RRP4 (pAC3656) or rrp4-G226D (pAC3659) LEU2 plasmid, and rrp404 mtr4A (ACY2540) cells containing the [MTR4; RRP40; URA3] (pAC3713) maintenance plasmid were transformed with RRP40 (pAC3652) or rrp40-W195R (pAC3655) LEU2 plasmid and selected on Ura- $\mathrm{Leu}^{-}$minimal media plates. Transformed cells containing both the URA3 maintenance plasmid and the RRP4/rrp4G226D or RRP40/rrp40-W195R LEU2 plasmid were subsequently transformed with MTR4 (pAC4096) or mtr4-F7A-F10A (pAC4099) HIS3 plasmids and selected on $\mathrm{Ura}^{-} \mathrm{Leu}^{-} \mathrm{His}^{-}$minimal media plates. The transformed cells were then streaked onto 5-FOA $\mathrm{Leu}^{-} \mathrm{His}^{-}$plates to select for cells that did not contain the URA3 maintenance plasmid. The resulting rrp4 $4 \mathrm{mtr} 4 \Delta$ cells containing only RRP4 or rrp4-G226D LEU2 plasmid and MTR4 or

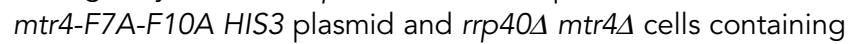
only RRP40 or rrp40-W195R LEU2 plasmid and MTR4 or mtr4F7A-F10A HIS3 plasmid were grown in $2 \mathrm{~mL} \mathrm{Leu}^{-} \mathrm{His}^{-}$minimal media overnight at $30^{\circ} \mathrm{C}$ to saturation, serially diluted, and spotted on $\mathrm{Leu}^{-} \mathrm{His}^{-}$minimal media plates. The plates were incubated at $30^{\circ} \mathrm{C}$ and $37^{\circ} \mathrm{C}$ for $3 \mathrm{~d}$.

\section{Coimmunoprecipitations}

All immunoprecipitations were performed using the same protocol. Cell samples were grown in $2 \mathrm{~mL}$ selective media overnight at $30^{\circ} \mathrm{C}$ to saturation and $10-20 \mathrm{~mL}$ cultures with an $\mathrm{OD}_{600}=0.2-0.3$ were prepared and grown at $30^{\circ} \mathrm{C}$ for $5 \mathrm{~h}$. Yeast cell lysates were prepared by resuspending cell pellets in $0.5-0.75 \mathrm{~mL}$ of IPP150 Buffer (10 mM Tris- $\mathrm{HCl}, \mathrm{pH}$ 8; $150 \mathrm{mM} \mathrm{NaCl}$; 0.1\% NP40, PMSF) supplemented with protease inhibitors (1 mM PMSF; Pierce Protease Inhibitors [Thermo Fisher Scientific]), and 300 $\mu \mathrm{L}$ of glass beads. Cells were disrupted in a Mini-Beadbeater 16 Cell Disrupter (BioSpec) for $4-5 \times 1 \mathrm{~min}$ at $25^{\circ} \mathrm{C}$ with $1 \mathrm{~min}$ on ice between repetitions. Crude lysate was transferred to a chilled microcentrifuge tube and remaining beads were washed with an additional $150 \mu \mathrm{L}$ of IPP150 Buffer. Lysate was then cleared by centrifugation at $16,000 \mathrm{~g}$ for $10 \mathrm{~min}$ at $4^{\circ} \mathrm{C}$. Protein lysate concentration was determined by Pierce BCA Protein Assay Kit (Life Technologies). For input samples, $40 \mu \mathrm{g}$ of cleared lysate was collected and frozen at $-20^{\circ} \mathrm{C}$. For coimmunoprecipitations, $1 \mathrm{mg}$ of cleared lysate in $1 \mathrm{~mL}$ IPP150 Buffer was prepared, $15-30 \mu \mathrm{L}$ of a 1:1 bead slurry of either Pierce Anti-c-Myc Magnetic Beads (Thermo Fisher) or IgG Sepharose 6 Fast Flow Beads (GE Healthcare) was added, and samples were incubated at $4^{\circ} \mathrm{C}$ 
overnight with mixing. After overnight incubation, beads were washed three times in $1 \mathrm{~mL}$ IPP150 Buffer for $15 \mathrm{sec}$ each (antiMyc beads) or 5 min each (lgG Sepharose beads). Whole cell lysate input samples $(40 \mu \mathrm{g})$ and total bound samples in reducing sample buffer were boiled for $5 \mathrm{~min}$ at $100^{\circ} \mathrm{C}$, resolved on $4 \%-$ 20\% Criterion TGX Stain-Free Precast Polyacrylamide Gels (BioRad), transferred to nitrocellulose membranes (Bio-Rad) and immunoblotted. Myc-tagged Rrp4 proteins were detected with mouse anti-Myc monoclonal antibody 9B11 (1:2000; Cell Signaling). TAP-tagged Rrp43 protein was detected with peroxidase anti-peroxidase (PAP) soluble complex antibody produced in rabbit (1:5000, Sigma-Aldrich). FLAG-tagged Mtr4 protein was detected with anti-DYKDDDDK (FLAG) tag rabbit monoclonal antibody D6W5B (1:2000; Cell Signaling). The 3-phosphoglycerate kinase (Pgk1) protein was detected using anti-Pgk1 monoclonal antibody (1:30,000; Invitrogen) as a loading control.

To assess association of Rrp4 G226D with the RNA exosome complex, we utilized RRP43-TAP (YCR035C) cells expressing RRP4-Myc (pAC3669), rrp4-G58V-Myc (pAC3670) or rrp4G226D-Myc (pAC3672) and RRP43-TAP rrp4A (ACY2803) cells expressing only RRP4-Myc (pAC3669) or rrp4-G226D-Myc (pAC3672) and immunoprecipitated Rrp43-TAP using the IgG Sepharose beads. Levels of associated Rrp4-Myc proteins with the Rrp43-TAP tagged subunit were detected by immunoblotting. To assess association of Rrp4 G226D with the Mtr4 helicase, we utilized rrp4A (yAV1103) cells expressing only RRP4-Myc (pAC3669) or rrp4-G226D-Myc (pAC3672) and coexpressing exogenous MTR4-FLAG (pAC3719) and immunoprecipitated Rrp4Myc or Rrp4 G226D-Myc using anti-c-Myc beads. Levels of associated Mtr4 with the Rrp4-Myc proteins were detected by immunoblotting.

\section{DATA DEPOSITION}

The raw RNA-seq data from this study have been submitted to the NCBI Gene Expression Omnibus (GEO) under accession GSE163106.

\section{SUPPLEMENTAL MATERIAL}

Supplemental material is available for this article.

\section{ACKNOWLEDGMENTS}

We thank members of the Corbett and van Hoof laboratories for critical discussions and input. We also thank Dr. Graeme Conn for his intellectual contributions. This work was supported by National Institutes of Health (NIH) R01 grants (GM058728) to A. H.C. and A.v.H. and GM099790 to A.v.H. M.C.S. was supported by a National Institute of General Medical Sciences (NIGMS) F31 grant (GM134649-01). L.E. was supported by the NIH-funded Emory Initiative for Maximizing Student Development (R25 GM099644). S.E.S. was supported by the National Science Foundation (NSF) Graduate Research Fellowship (GRFP 1937971). M.A.B is supported by the NIH-funded Intramural Research Program of the National Cancer Institute. Lastly, we would also like to thank the RNA society and Saccharomyces
Genomic Database (SGD) (Cherry et al. 2012) for providing community resources and support for scientific discovery.

Received December 11, 2020; accepted June 11, 2021.

\section{REFERENCES}

Adams A, Gottschling DE, Kaiser CA, Stearns T. 1997. Methods in yeast genetics. Cold Spring Harbor Laboratory Press, Cold Spring Harbor, NY.

Adzhubei IA, Schmidt S, Peshkin L, Ramensky VE, Gerasimova A, Bork P, Kondrashov AS, Sunyaev SR. 2010. A method and server for predicting damaging missense mutations. Nat Methods 7: 248-249. doi:10.1038/nmeth0410-248

Allmang C, Kufel J, Chanfreau G, Mitchell P, Petfalski E, Tollervey D. 1999. Functions of the exosome in rRNA, snoRNA and snRNA synthesis. EMBO J 18: 5399-5410. doi:10.1093/emboj/18.19.5399

Belair C, Sim S, Wolin SL. 2018. Noncoding RNA surveillance: the ends justify the means. Chem Rev 118: 4422-4447. doi:10 .1021/acs.chemrev.7b00462

Belair C, Sim S, Kim KY, Tanaka Y, Park IH, Wolin SL. 2019. The RNA exosome nuclease complex regulates human embryonic stem cell differentiation. J Cell Biol 218: 2564-2582. doi:10.1083/jcb .201811148

Biancheri R, Cassandrini D, Pinto F, Trovato R, Di Rocco M, MirabelliBadenier M, Pedemonte M, Panicucci C, Trucks H, Sander T, et al. 2013. EXOSC3 mutations in isolated cerebellar hypoplasia and spinal anterior horn involvement. J Neurol 260: 1866-1870. doi:10.1007/s00415-013-6896-0

Boczonadi V, Mueller JS, Pyle A, Munkley J, Dor T, Quartararo J, Ferrero I, Karcagi V, Giunta M, Polvikoski T, et al. 2014. EXOSC8 mutations alter mRNA metabolism and cause hypomyelination with spinal muscular atrophy and cerebellar hypoplasia. Nat Commun 5: 4287. doi:10.1038/ncomms5287

Bonneau F, Basquin J, Ebert J, Lorentzen E, Conti E. 2009. The yeast exosome functions as a macromolecular cage to channel RNA substrates for degradation. Cell 139: 547-559. doi:10.1016/j.cell .2009.08.042

Briggs MW, Burkard KT, Butler JS. 1998. Rrp6p, the yeast homologue of the human PM-Scl 100-kDa autoantigen, is essential for efficient 5.8 S rRNA 3' end formation. J Biol Chem 273: 13255-13263. doi:10.1074/jbc.273.21.13255

Burke D, Dawson D, Stearns T. 2000. Methods in yeast genetics: a Cold Spring Harbor Laboratory course manual. Cold Spring Harbor Laboratory Press, Cold Spring Harbor, NY.

Burns D, Donkervoort D, Bharucha-Goebel D, Giunta M, Munro B, Scavina M, Foley R, Müller J, Bönnemann C, Horvath R. 2017. A recessive mutation in EXOSC9 causes abnormal RNA metabolism resulting in a novel form of cerebellar hypoplasia/atrophy with early motor neuronopathy. Neuromuscul Disord 27: S38. doi:10 .1016/S0960-8966(17)30331-0

Burns DT, Donkervoort S, Muller JS, Knierim E, Bharucha-Goebel D, Faqeih EA, Bell SK, AlFaifi AY, Monies D, Millan F, et al. 2018. Variants in EXOSC9 disrupt the RNA exosome and result in cerebellar atrophy with spinal motor neuronopathy. Am J Hum Genet 102: 858-873. doi:10.1016/j.ajhg.2018.03.011

Chen EY, Tan CM, Kou Y, Duan Q, Wang Z, Meirelles GV, Clark NR, Ma'ayan A. 2013. Enrichr: interactive and collaborative HTML5 gene list enrichment analysis tool. BMC Bioinformatics 14: 128. doi:10.1186/1471-2105-14-128

Cherry JM, Hong EL, Amundsen C, Balakrishnan R, Binkley G, Chan ET, Christie KR, Costanzo MC, Dwight SS, Engel SR, et al. 2012. Saccharomyces Genome Database: the genomics resource 
of budding yeast. Nucleic Acids Res 40: D700-D705. doi:10.1093/ nar/gkr1029

Choi Y. 2012. A fast computation of pairwise sequence alignment scores between a protein and a set of single-locus variants of another protein. In Proceedings of the ACM conference on bioinformatics, computational biology and biomedicine, pp. 414-417. Association for Computing Machinery, Orlando, Florida.

Choi Y, Sims GE, Murphy S, Miller JR, Chan AP. 2012. Predicting the functional effect of amino acid substitutions and indels. PLoS One 7: e46688. doi:10.1371/journal.pone.0046688

Coy S, Volanakis A, Shah S, Vasiljeva L. 2013. The Sm complex is required for the processing of non-coding RNAs by the exosome. PLoS One 8: e65606. doi:10.1371/journal.pone.0065606

Davis CA, Ares M Jr. 2006. Accumulation of unstable promoter-associated transcripts upon loss of the nuclear exosome subunit Rrp6p in Saccharomyces cerevisiae. Proc Natl Acad Sci 103: 3262-3267. doi:10.1073/pnas.0507783103

de Amorim J, Slavotinek A, Fasken MB, Corbett AH, Morton DJ. 2020. Modeling pathogenic variants in the RNA exosome. RNA Dis 7: e1166.

de la Cruz J, Kressler D, Tollervey D, Linder P. 1998. Dob1p (Mtr4p) is a putative ATP-dependent RNA helicase required for the $3^{\prime}$ end formation of 5.8S rRNA in Saccharomyces cerevisiae. EMBO J 17: 1128-1140. doi:10.1093/emboj/17.4.1128

Delan-Forino C, Schneider C, Tollervey D. 2017. Transcriptome-wide analysis of alternative routes for RNA substrates into the exosome complex. PLoS Genet 13: e1006699. doi:10.1371/journal.pgen .1006699

Di Donato N, Neuhann T, Kahlert A-K, Klink B, Hackmann K, Neuhann I, Novotna B, Schallner J, Krause C, Glass IA, et al. 2016. Mutations in EXOSC2 are associated with a novel syndrome characterised by retinitis pigmentosa, progressive hearing loss, premature ageing, short stature, mild intellectual disability and distinctive gestalt. J Med Genet 53: 419-425. doi:10.1136/jmedg enet-2015-103511

Dobin A, Davis CA, Schlesinger F, Drenkow J, Zaleski C, Jha S, BatutP, Chaisson M, Gingeras TR. 2012. STAR: ultrafast universal RNA-seq aligner. Bioinformatics 29: 15-21. doi:10.1093/bioinformatics/ bts635

Donahue TF, Henry SA. 1981. myo-Inositol-1-phosphate synthase. Characteristics of the enzyme and identification of its structural gene in yeast. J Biol Chem 256: 7077-7085. doi:10.1016/ S0021-9258(19)69102-7

Eggens VRC, Barth PG, Niermeijer J-MF, Berg JN, Darin N, Dixit A, Fluss J, Foulds N, Fowler D, Hortobágyi T, et al. 2014. EXOSC3 mutations in pontocerebellar hypoplasia type 1: novel mutations and genotype-phenotype correlations. Orphanet J Rare Dis 9: 23. doi:10.1186/1750-1172-9-23

Falk S, Bonneau F, Ebert J, Kogel A, Conti E. 2017. Mpp6 incorporation in the nuclear exosome contributes to RNA channeling through the Mtr4 helicase. Cell Rep 20: 2279-2286. doi:10 .1016/j.celrep.2017.08.033

Fasken MB, Leung SW, Banerjee A, Kodani MO, Chavez R, Bowman EA, Purohit MK, Rubinson ME, Rubinson EH, Corbett AH. 2011. Air1 zinc knuckles 4 and 5 and a conserved IWRXY motif are critical for the function and integrity of the Trf4/ 5-Air1/2-Mtr4 polyadenylation (TRAMP) RNA quality control complex. J Biol Chem 286: 37429-37445. doi:10.1074/jbc.M111 .271494

Fasken MB, Losh JS, Leung SW, Brutus S, Avin B, Vaught JC, PotterBirriel J, Craig T, Conn GL, Mills-Lujan K, et al. 2017. Insight into the RNA exosome complex through modeling pontocerebellar hypoplasia type $1 \mathrm{~b}$ disease mutations in yeast. Genetics 205: 221237. doi:10.1534/genetics.116.195917
Fasken MB, Morton DJ, Kuiper EG, Jones SK, Leung SW, Corbett AH. 2020. The RNA exosome and human disease. Methods Mol Biol 2062: 3-33. doi:10.1007/978-1-4939-9822-7_1

Fox MJ, Gao H, Smith-Kinnaman WR, Liu Y, Mosley AL. 2015. The exosome component Rrp6 is required for RNA polymerase II termination at specific targets of the Nrd1-Nab3 pathway. PLoS Genet 11: e1004999. doi:10.1371/journal.pgen.1004999

Ghaemmaghami S, Huh WK, Bower K, Howson RW, Belle A, Dephoure N, O'Shea EK, Weissman JS. 2003. Global analysis of protein expression in yeast. Nature 425: 737-741. doi:10.1038/ nature02046

Gillespie A, Gabunilas J, Jen JC, Chanfreau GF. 2017. Mutations of EXOSC3/Rrp40p associated with neurological diseases impact ribosomal RNA processing functions of the exosome in S. cerevisiae. RNA 23: 466-472. doi:10.1261/rna.060004.116

Gudipati RK, Xu Z, Lebreton A, Seraphin B, Steinmetz LM, Jacquier A, Libri D. 2012. Extensive degradation of RNA precursors by the exosome in wild-type cells. Mol Cell 48: 409-421. doi:10.1016/j .molcel.2012.08.018

Halevy A, Lerer I, Cohen R, Kornreich L, Shuper A, Gamliel M, Zimerman BE, Korabi I, Meiner V, Straussberg R, et al. 2014. Novel EXOSC3 mutation causes complicated hereditary spastic paraplegia. J Neurol 261: 2165-2169. doi:10.1007/s00415-0147457-x

Hecht M, Bromberg Y, Rost B. 2015. Better prediction of functional effects for sequence variants. BMC Genomics 16: S1. doi:10.1186/ 1471-2164-16-S8-S1

Hou D, Ruiz M, Andrulis ED. 2012. The ribonuclease Dis3 is an essential regulator of the developmental transcriptome. BMC Genomics 13: 359. doi:10.1186/1471-2164-13-359

Houseley J, Tollervey D. 2008. The nuclear RNA surveillance machinery: the link between ncRNAs and genome structure in budding yeast? Biochim Biophys Acta 1779: 239-246. doi:10.1016/j .bbagrm.2007.12.008

Houseley J, Tollervey D. 2009. The many pathways of RNA degradation. Cell 136: 763-776. doi:10.1016/j.cell.2009.01.019

Kilchert C, Wittmann S, Vasiljeva L. 2016a. The regulation and functions of the nuclear RNA exosome complex. Nat Rev Mol Cell Biol 17: 227-239. doi:10.1038/nrm.2015.15

Kilchert C, Wittmann S, Vasiljeva L. 2016b. The regulation and functions of the nuclear RNA exosome complex. Nat Rev Mol Cell Biol 17: 227-239. doi:10.1038/nrm.2015.15

Klig LS, Henry SA. 1984. Isolation of the yeast INO1 gene: located on an autonomously replicating plasmid, the gene is fully regulated. Proc Natl Acad Sci 81: 3816-3820. doi:10.1073/pnas.81.12.3816

Kuleshov MV, Jones MR, Rouillard AD, Fernandez NF, Duan Q, Wang Z, Koplev S, Jenkins SL, Jagodnik KM, Lachmann A, et al. 2016. Enrichr: a comprehensive gene set enrichment analysis web server 2016 update. Nucleic Acids Res 44: W90-W97. doi:10.1093/nar/gkw377

Kuleshov MV, Diaz JEL, Flamholz ZN, Keenan AB, Lachmann A, Wojciechowicz ML, Cagan RL, Ma'ayan A. 2019. modEnrichr: a suite of gene set enrichment analysis tools for model organisms. Nucleic Acids Res 47: W183-W190. doi:10.1093/nar/gkz347

LaCava J, Houseley J, Saveanu C, Petfalski E, Thompson E, Jacquier A, Tollervey D. 2005. RNA degradation by the exosome is promoted by a nuclear polyadenylation complex. Cell 121: 713724. doi:10.1016/j.cell.2005.04.029

Liao Y, Smyth GK, Shi W. 2014. featureCounts: an efficient general purpose program for assigning sequence reads to genomic features. Bioinformatics 30: 923-930. doi:10.1093/bioinformatics/ btt656

Lim SJ, Boyle PJ, Chinen M, Dale RK, Lei EP. 2013. Genome-wide localization of exosome components to active promoters and 
chromatin insulators in Drosophila. Nucleic Acids Res 41: 2963 2980. doi:10.1093/nar/gkt037

Liu Q, Greimann JC, Lima CD. 2006. Reconstitution, activities, and structure of the eukaryotic RNA exosome. Cell 127: 1223-1237. doi:10.1016/j.cell.2006.10.037

Livak KJ, Schmittgen TD. 2001. Analysis of relative gene expression data using real-time quantitative $P C R$ and the $2^{-\Delta \Delta C}$ method. Methods 25: 402-408. doi:10.1006/meth.2001.1262

Lorentzen E, Dziembowski A, Lindner D, Seraphin B, Conti E. 2007. RNA channelling by the archaeal exosome. EMBO Rep 8: 470476. doi:10.1038/sj.embor.7400945

Losh J. 2018. "Identifying subunit organization and function of the nuclear RNA exosome machinery." PhD thesis, The University of Texas Health Science Center at Houston.

Love MI, Huber W, Anders S. 2014. Moderated estimation of fold change and dispersion for RNA-seq data with DESeq2. Genome Biol 15: 550. doi:10.1186/s13059-014-0550-8

Lubas M, Christensen MS, Kristiansen MS, Domanski M, Falkenby LG, Lykke-Andersen S, Andersen JS, Dziembowski A, Jensen TH. 2011. Interaction profiling identifies the human nuclear exosome targeting complex. Mol Cell 43: 624-637. doi:10.1016/j.molcel .2011.06.028

Makino DL, Baumgaertner M, Conti E. 2013. Crystal structure of an RNA-bound 11-subunit eukaryotic exosome complex. Nature 495: 70-75. doi:10.1038/nature11870

Marquardt S, Hazelbaker DZ, Buratowski S. 2011. Distinct RNA degradation pathways and $3^{\prime}$ extensions of yeast non-coding RNA species. Transcription 2: 145-154. doi:10.4161/trns.2.3.16298

Mitchell P, Petfalski E, Tollervey D. 1996. The $3^{\prime}$ end of yeast $5.8 \mathrm{~S}$ rRNA is generated by an exonuclease processing mechanism. Genes Dev 10: 502-513. doi:10.1101/gad.10.4.502

Mitchell P, Petfalski E, Shevchenko A, Mann M, Tollervey D. 1997. The exosome: a conserved eukaryotic RNA processing complex containing multiple $3^{\prime} \rightarrow 5^{\prime}$ exoribonucleases. Cell 91: 457-466. doi:10.1016/S0092-8674(00)80432-8

Mitchell P, Petfalski E, Houalla R, Podtelejnikov A, Mann M, Tollervey D. 2003. Rrp47p is an exosome-associated protein required for the $3^{\prime}$ processing of stable RNAs. Mol Cell Biol 23: 6982-6992. doi:10.1128/MCB.23.19.6982-6992.2003

Moore MJ, Proudfoot NJ. 2009. Pre-mRNA processing reaches back to transcription and ahead to translation. Cell 136: 688-700. doi:10.1016/j.cell.2009.02.001

Morton DJ, Kuiper EG, Jones SK, Leung SW, Corbett AH, Fasken MB. 2018. The RNA exosome and RNA exosome-linked disease. RNA 24: 127-142. doi:10.1261/rna.064626.117

Morton DJ, Jalloh B, Kim L, Kremsky I, Nair RJ, Nguyen KB, Rounds JC, Sterrett MC, Brown B, Le T, et al. 2020. A Drosophila model of Pontocerebellar Hypoplasia reveals a critical role for the RNA exosome in neurons. PLoS Genet 16: e1008901. doi:10.1371/journal.pgen.1008901

Oddone A, Lorentzen E, Basquin J, Gasch A, Rybin V, Conti E, Sattler M. 2007. Structural and biochemical characterization of the yeast exosome component Rrp40. EMBO Rep 8: 63-69. doi:10.1038/sj.embor.7400856

Parker R. 2012. RNA degradation in Saccharomyces cerevisae. Genetics 191: 671-702. doi:10.1534/genetics.111.137265

Pefanis E, Wang J, Rothschild G, Lim J, Chao J, Rabadan R, Economides AN, Basu U. 2014. Noncoding RNA transcription targets AID to divergently transcribed loci in B cells. Nature 514: 389-393. doi:10.1038/nature13580

Rodrigues CHM, Myung Y, Pires DEV, Ascher DB. 2019. mCSM-PPI2: predicting the effects of mutations on protein-protein interactions. Nucleic Acids Res 47: W338-W344. doi:10.1093/nar/ gkz383
Rodríguez-Galán O, García-Gómez JJ, Kressler D, de la Cruz J. 2015. Immature large ribosomal subunits containing the $7 \mathrm{~S}$ pre-rRNA can engage in translation in Saccharomyces cerevisiae. RNA Biol 12: 838-846. doi:10.1080/15476286.2015.1058477

Sambrook J, Fritsch EF, Maniatis T. 1989. Molecular cloning: a laboratory manual. Cold Spring Harbor Laboratory Press, Cold Spring Harbor, NY.

Schaeffer D, Tsanova B, Barbas A, Reis FP, Dastidar EG, SanchezRotunno M, Arraiano CM, van Hoof A. 2009. The exosome contains domains with specific endoribonuclease, exoribonuclease and cytoplasmic mRNA decay activities. Nat Struct Mol Biol 16: 56-62. doi:10.1038/nsmb.1528

Schneider C, Tollervey D. 2013. Threading the barrel of the RNA exosome. Trends Biochem Sci 38: 485-493. doi:10.1016/j.tibs.2013 .06 .013

Schneider C, Kudla G, Wlotzka W, Tuck A, Tollervey D. 2012. Transcriptome-wide analysis of exosome targets. Mol Cell 48: 422-433. doi:10.1016/j.molcel.2012.08.013

Schottmann G, Picker-Minh S, Schwarz J, Gill E, Rodenburg R, Stenzel W, Kaindl A M, Schuelke M. 2017. Recessive mutation in EXOSC3 associates with mitochondrial dysfunction and pontocerebellar hypoplasia. Mitochondrion 37: 46-54. doi:10.1016/j.mito .2017.06.007

Schuch B, Feigenbutz M, Makino DL, Falk S, Basquin C, Mitchell P, Conti E. 2014. The exosome-binding factors Rrp6 and Rrp47 form a composite surface for recruiting the Mtr4 helicase. EMBO J 33: 2829-2846. doi:10.15252/embj.201488757

Schuller JM, Falk S, Fromm L, Hurt E, Conti E. 2018. Structure of the nuclear exosome captured on a maturing preribosome. Science 360: 219-222. doi:10.1126/science.aar5428

Sikorski RS, Hieter P. 1989. A system of shuttle vectors and yeast host strains designed for efficient manipulation of DNA in Saccharomyces cerevisiae. Genetics 122: 19-27. doi:10.1093/ge netics/122.1.19

Slavotinek A, Misceo D, Htun S, Mathisen L, Frengen E, Foreman M, Hurtig JE, Enyenihi L, Sterrett MC, Leung SW, et al. 2020. Biallelic variants in the RNA exosome gene EXOSC5 are associated with developmental delays, short stature, cerebellar hypoplasia and motor weakness. Hum Mol Genet 29: 2218-2239. doi:10 $.1093 / \mathrm{hmg} /$ ddaa108

Steinmetz EJ, Brow DA. 1998. Control of pre-mRNA accumulation by the essential yeast protein Nrd1 requires high-affinity transcript binding and a domain implicated in RNA polymerase II association. Proc Natl Acad Sci 95: 6699-6704. doi:10.1073/pnas.95.12 .6699

Steinmetz EJ, Conrad NK, Brow DA, Corden JL. 2001. RNA-binding protein $\mathrm{Nrd} 1$ directs poly(A)-independent 3 '-end formation of RNA polymerase II transcripts. Nature 413: 327-331. doi:10 $.1038 / 35095090$

Stuparevic I, Mosrin-Huaman C, Hervouet-Coste N, Remenaric M, Rahmouni AR. 2013. Cotranscriptional recruitment of RNA exosome cofactors Rrp47p and Mpp6p and two distinct Trf-Air-Mtr4 polyadenylation (TRAMP) complexes assists the exonuclease Rrp6p in the targeting and degradation of an aberrant messenger ribonucleoprotein particle (mRNP) in yeast. J Biol Chem 288: 31816-31829. doi:10.1074/jbc.M113.491290

Vaňáčová Š, Wolf J, Martin G, Blank D, Dettwiler S, Friedlein A, Langen $\mathrm{H}$, Keith $\mathrm{G}$, Keller W. 2005. A new yeast poly(A) polymerase complex involved in RNA quality control. PLoS Biol 3: e189. doi:10.1371/journal.pbio.0030189

van Hoof A, Lennertz P, Parker R. 2000. Yeast exosome mutants accumulate 3 '-extended polyadenylated forms of U4 small nuclear RNA and small nucleolar RNAs. Mol Cell Biol 20: 441-452. doi:10.1128/MCB.20.2.441-452.2000 
Wan J, Yourshaw M, Mamsa H, Rudnik-Schoeneborn S, Menezes MP, Hong JE, Leong DW, Senderek J, Salman MS, Chitayat D, et al. 2012. Mutations in the RNA exosome component gene EXOSC3 cause pontocerebellar hypoplasia and spinal motor neuron degeneration. Nat Genet 44: 704-U134. doi:10.1038/ng.2254

Wasmuth EV, Januszyk K, Lima CD. 2014. Structure of an Rrp6-RNA exosome complex bound to poly(A) RNA. Nature 511: 435-439. doi:10.1038/nature13406

Wasmuth EV, Zinder JC, Zattas D, Das M, Lima CD. 2017. Structure and reconstitution of yeast Mpp6-nuclear exosome complexes reveals that Mpp6 stimulates RNA decay and recruits the Mtr4 helicase. Elife 6: 24. doi:10.7554/eLife.29062

Weick EM, Puno MR, Januszyk K, Zinder JC, DiMattia MA, Lima CD. 2018. Helicase-dependent RNA decay illuminated by a cryo-EM structure of a human nuclear RNA exosome-MTR4 complex. Cell 173: 1663-1677.e1621. doi:10.1016/j.cell.2018.05.041

Wolin SL, Sim S, Chen X. 2012. Nuclear noncoding RNA surveillance: is the end in sight? Trends Genet 28: 306-313. doi:10.1016/j.tig .2012.03.005

Wyers $F$, Rougemaille $M$, Badis $G$, Rousselle J-C, Dufour $M-E$, Boulay J, Régnault B, Devaux F, Namane A, Séraphin B, et al. 2005. Cryptic Pol II transcripts are degraded by a nuclear quality control pathway involving a new poly(A) polymerase. Cell 121: 725-737. doi:10.1016/j.cell.2005.04.030

Xu Z, Wei W, Gagneur J, Perocchi F, Clauder-Münster S, Camblong J, Guffanti E, Stutz F, Huber W, Steinmetz LM. 2009. Bidirectional promoters generate pervasive transcription in yeast. Nature 457: 1033-1037. doi:10.1038/nature07728

Yang X, Bayat V, DiDonato N, Zhao Y, Zarnegar B, Siprashvili Z, LopezPajares V, Sun T, Tao S, Li C, et al. 2019. Genetic and genomic studies of pathogenic EXOSC2 mutations in the newly described disease SHRF implicate the autophagy pathway in disease pathogenesis. Hum Mol Genet 29: 541-553. doi:10.1093/hmg/ddz251

Yatsuka H, Hada K, Shiraishi H, Umeda R, Morisaki I, Urushibata H, Shimizu N, Sebastian WA, Hikida T, Ishitani T, et al. 2020. Exosc2 deficiency leads to developmental disorders by causing a nucleotide pool imbalance in zebrafish. Biochem Biophys Res Commun.

Zinder JC, Lima CD. 2017. Targeting RNA for processing or destruction by the eukaryotic RNA exosome and its cofactors. Genes Dev 31: 88-100. doi:10.1101/gad.294769.116

Zinder JC, Wasmuth EV, Lima CD. 2016. Nuclear RNA exosome at 3.1 Å reveals substrate specificities, RNA paths, and allosteric inhibition of Rrp44/Dis3. Mol Cell 64: 734-745. doi:10.1016/j.molcel .2016.09.038 

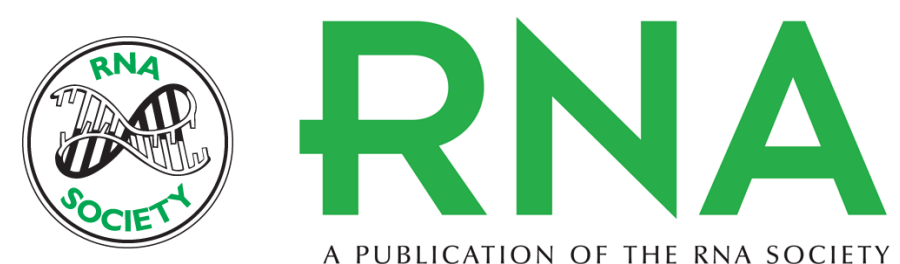

A PUBLICATION OF THE RNA SOCIETY

\title{
A budding yeast model for human disease mutations in the EXOSC2 cap subunit of the RNA exosome complex
}

\author{
Maria C. Sterrett, Liz Enyenihi, Sara W. Leung, et al.
}

RNA 2021 27: 1046-1067 originally published online June 23, 2021

Access the most recent version at doi:10.1261/rna.078618.120

\section{Supplemental http://rnajournal.cshlp.org/content/suppl/2021/06/23/rna.078618.120.DC1 Material}

References This article cites 84 articles, 22 of which can be accessed free at: http://rnajournal.cshlp.org/content/27/9/1046.full.html\#ref-list-1

Open Access Freely available online through the RNA Open Access option.

Creative This article, published in RNA, is available under a Creative Commons License Commons (Attribution 4.0 International), as described at

License http://creativecommons.org/licenses/by/4.0/.

Email Alerting Receive free email alerts when new articles cite this article - sign up in the box at the Service top right corner of the article or click here.

\section{|||||||| Providing Precise Solutions for your research.}

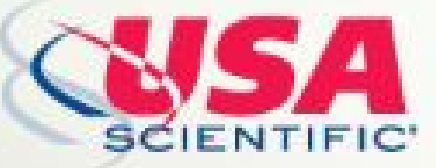

To subscribe to RNA go to:

http://rnajournal.cshlp.org/subscriptions

(C) 2021 Sterrett et al.; Published by Cold Spring Harbor Laboratory Press for the RNA Society 